\title{
Magnetic small-angle neutron scattering on bulk metallic glasses: A feasibility study for imaging displacement fields
}

\author{
Denis Mettus, ${ }^{1,}$ Michael Deckarm, ${ }^{2}$ Andreas Leibner, ${ }^{2}$ Rainer Birringer,${ }^{2, \dagger}$ Moritz Stolpe,${ }^{3}$ Ralf Busch, ${ }^{3}$ Dirk Honecker, ${ }^{4}$ \\ Joachim Kohlbrecher, ${ }^{5}$ Patrick Hautle, ${ }^{5}$ Nemanja Niketic, ${ }^{5}$ Jesús Rodríguez Fernández, ${ }^{6}$ \\ Luis Fernández Barquín, ${ }^{6}$ and Andreas Michels ${ }^{1, \ddagger}$ \\ ${ }^{1}$ Physics and Materials Science Research Unit, University of Luxembourg, 162A Avenue de la Fä̈encerie, L-1511 Luxembourg, \\ Grand Duchy of Luxembourg \\ ${ }^{2}$ Universität des Saarlandes, Experimentalphysik Campus D2 2, D-66123, Saarbrücken, Germany \\ ${ }^{3}$ Universität des Saarlandes, Chair of Metallic Materials, Department of Materials Science and Engineering Campus C6.3, \\ D-66123 Saarbrücken, Germany \\ ${ }^{4}$ Institut Laue-Langevin, 6 Rue Jules Horowitz, Boîte Postale 156, F-38042 Grenoble Cedex 9, France \\ ${ }^{5}$ Paul Scherrer Institute, CH-5232 Villigen PSI, Switzerland \\ ${ }^{6}$ CITIMAC, Universidad de Cantabria, 39005 Santander, Spain \\ (Received 28 September 2017; published 11 December 2017)
}

\begin{abstract}
Magnetic-field-dependent small-angle neutron scattering (SANS) has been utilized to study the magnetic microstructure of bulk metallic glasses (BMGs). In particular, the magnetic scattering from soft magnetic $\mathrm{Fe}_{70} \mathrm{Mo}_{5} \mathrm{Ni}_{5} \mathrm{P}_{12.5} \mathrm{~B}_{2.5} \mathrm{C}_{5}$ and hard magnetic $\left(\mathrm{Nd}_{60} \mathrm{Fe}_{30} \mathrm{Al}_{10}\right)_{92} \mathrm{Ni}_{8}$ alloys in the as-prepared, aged, and mechanically deformed state is compared. While the soft magnetic BMGs exhibit a large field-dependent SANS response with perturbations originating predominantly from spatially varying magnetic anisotropy fields, the SANS cross sections of the hard magnetic BMGs are only weakly dependent on the field, and their angular anisotropy indicates the presence of scattering contributions due to spatially dependent saturation magnetization. Moreover, we observe an unusual increase in the magnetization of the rare-earth-based alloy after deformation. Analysis of the SANS cross sections in terms of the correlation function of the spin misalignment reveals the existence of field-dependent anisotropic long-wavelength magnetization fluctuations on a scale of a few tens of nanometers. We also give a detailed account of how the SANS technique relates to unraveling displacement fields on a mesoscopic length scale in disordered magnetic materials.
\end{abstract}

DOI: 10.1103/PhysRevMaterials.1.074403

\section{INTRODUCTION}

Bulk metallic glasses (BMGs) are amorphous solids that are well known for their excellent mechanical properties, which render them attractive for many technological applications [1-4]. Although these materials are heavily investigated both experimentally and theoretically, the yielding and failure of BMGs under external strain are still not fully understood. In particular, the discrete nature of the flow defect and/or the event in amorphous solids, which enables inelastic deformation [5] before runaway shear bands form leading to catastrophic failure, is still a critical issue.

Recently, Dasgupta, Hentschel, and Procaccia [6,7] carried out numerical simulations and analytical theory to discover that upon applying external simple shear, localized plastic instabilities occur before the onset of macroscopic yielding. The nature of these plastic events has been identified as isolated Eshelby quadrupoles that organize the nonaffine displacement field around a localized shear transformation. Indeed, the quadrupolar character of the displacement field has been verified experimentally by means of shear-deformation experiments on a monodisperse hard-sphere colloidal glass using confocal microscopy [8].

\footnotetext{
*denis.mettus@uni.lu

†r.birringer@nano.uni-saarland.de

†andreas.michels@uni.lu
}

To establish the context for the later discussion of the magnetic neutron scattering data of the amorphous BMG samples, we refer to the early work of Kronmüller, Seeger, and Wilkens $[9,10]$, who pioneered the use of the small-angle neutron scattering (SANS) technique for studying spin disorder due to the strain fields of dislocations in crystalline materials: in mechanically deformed metals, the magnetization is highly inhomogeneous in the vicinity of dislocations, which is due to the presence of magnetoelastic coupling. The associated static long-wavelength magnetization fluctuations represent a contrast for elastic magnetic SANS, and they can be computed by means of the continuum theory of micromagnetics for samples close to magnetic saturation. Most importantly, this type of magnetic SANS-denoted as spin-misalignment scattering - has been predicted to be about 10-100 times larger than the nuclear SANS that is related to the volume dilatations of dislocations [9]. Although a dislocation is defined with reference to an ordered crystalline lattice, both conceptsdislocations as well as shear transformations in disordered materials - are described using elastic continuum theory. We therefore conjecture that the ideas of Kronmüller, Seeger, and Wilkens can be adapted to the study of displacement fields in magnetic BMGs.

The mechanisms by which spin disorder in magnetic materials is generated are essentially related to (i) magnetoelastic and magnetocrystalline anisotropy, and (ii) internal magnetostatic stray fields, for instance due to spatially fluctuating materials parameters. To be more specific, magnetic forces generated by the distortion of the crystal lattice in the vicinity 
of a defect tend to modify the local magnetization vector field due to magnetoelastic coupling, while magnetocrystalline anisotropy tries to pull the magnetic moments along the principal axes of the crystal [11]. Likewise, jumps in the values of magnetization, exchange, or anisotropy constants at internal interfaces (e.g., in a magnetic nanocomposite or nanoporous ferromagnet) give rise to inhomogeneous magnetization states, which contribute significantly to the magnetic SANS signal. It is also important to emphasize that the adjustment of the magnetization along the respective local "easy" axes does not occur abruptly, i.e., on a scale of the interatomic spacing, rather it requires a more extended range. This is a consequence of the quantum-mechanical exchange interaction, which spreads out local perturbations in the magnetization over larger distances. The size of such spin inhomogeneities is characterized by a micromagnetic exchange length, which varies continuously with the applied field and takes on values between about 1-100 nm, a size regime that is routinely accessible by the SANS technique.

For the case of magnetic BMG alloys, which are studied in the present paper, it may be expected that the displacement field couples to the magnetization distribution $\mathbf{M}(\mathbf{r})$ by virtue of the magnetoelastic coupling energy, which links the components of the strain tensor $\epsilon_{i j}$ to $\mathbf{M}$; in other words, the spins might decorate the spatial distribution of the displacement field. Since the spatial extent of these displacement fields (quadrupolar structures) is of the order of a few $10 \mathrm{~nm}$, the SANS technique is ideally suited to visualize the associated spin structures in the bulk of the material; in fact, we are not aware of any other method that can accomplish this. Moreover, the application of an external magnetic field allows one to tune the size of the spin-misaligned regions, since the characteristic wavelength of the spin-misalignment fluctuations is expected to decrease with increasing field [12].

In the present paper, we discuss the influence of mechanical deformation on the magnetic microstructure of a soft and hard magnetic BMG by means of magnetic-field-dependent SANS. The choice of these materials enables one to explore-in the spirit of a feasibility study - the limiting cases of weak and strong magnetic anisotropy and, hence, different degrees of magnetoelastic coupling of displacement to magnetization. We present and compare the results of unpolarized SANS measurements, and we analyze their SANS cross sections in real space by computing the correlation function of the spin misalignment. Finally, we discuss how the expected scattering signal of displacement fields can be separated from other scattering contributions. We also refer to the supplemental material, which shows additional neutron data [13].

\section{EXPERIMENT}

The SANS experiments were carried at the instruments D33 [14,15] at the Institut Laue-Langevin (ILL), Grenoble, France and at SANS-I [16] at the Paul Scherrer Institute (PSI), Villingen, Switzerland. We used unpolarized incident neutrons with a mean wavelength of $\lambda=6 \AA$ and a wavelength broadening of $\Delta \lambda / \lambda \sim 10 \%$ [full width at half-maximum (FWHM)]. The instruments were set to cover a $q$-range of $0.035 \lesssim q \lesssim$ $1.5 \mathrm{~nm}^{-1}$. Neutron-scattering data were recorded at room temperature as a function of an external magnetic field $\mathbf{H}_{0}$,

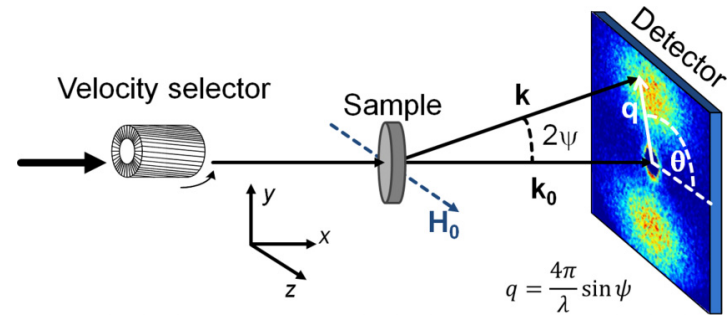

FIG. 1. Sketch of the perpendicular scattering SANS geometry, where the applied magnetic field $\mathbf{H}_{0}$ is perpendicular to the wave vector $\mathbf{k}_{0}$ of the incoming neutron beam. The scattering vector $\mathbf{q}$ is defined as the difference between the wave vectors of the scattered $\mathbf{k}$ and incident $\mathbf{k}_{0}$ neutrons. The mean wavelength $\lambda$ of the neutrons is selected by the velocity selector; $2 \psi$ is the scattering angle, and $\theta$ is the angle between $\mathbf{H}_{0}$ and $\mathbf{q}$ in the plane of the detector.

which was applied perpendicular to the wave vector $\mathbf{k}_{0}$ of the incident neutron beam $\left(\mathbf{k}_{0} \perp \mathbf{H}_{0}\right)$; see Fig. 1 for a sketch of the SANS setup. The field was cycled by first applying a large value, which is assumed to bring the sample into the approach-to-saturation regime, and then decreasing it following the major hysteresis loop. SANS-data reduction (correction for background scattering, transmission, detector efficiency) was carried out using the GRASP software package [17]. The transmission of all samples was measured at each applied-field value and found to be $\sim 79-85 \%$ for the soft magnetic BMG and 76-82\% for the hard magnetic BMG (see Fig. 8 in the Supplemental Material [13]).

The soft magnetic samples with the nominal atomic composition $\mathrm{Fe}_{70} \mathrm{Mo}_{5} \mathrm{Ni}_{5} \mathrm{P}_{12.5} \mathrm{~B}_{2.5} \mathrm{C}_{5}$ were produced by Glassimetal Technology, Inc. From the as-cast cylinder with a $4 \mathrm{~mm}$ diameter, three disks with thicknesses of, respectively, 0.36 , 0.38 , and $0.28 \mathrm{~mm}$ were cut. One sample remained in the as-cast state, one specimen was aged at a temperature of $358^{\circ} \mathrm{C}$ for $8 \mathrm{~h}$ in $\mathrm{N}_{2}$ atmosphere, and one disk was mechanically deformed. Mechanical deformation required two flat and parallel surfaces in order to apply compressive stress. Therefore, the cylindrical-shaped specimen was truncated by grinding to obtain a cross section defined by two parallel second lines with a distance of $3.66 \mathrm{~mm}$. After deformation this distance shrank to $3.60 \mathrm{~mm}$, resulting in an engineering strain of $\approx 1.6 \%$. We selected this deformation mode to induce a change of state of stress across the plane of the specimen (compressive stress parallel and tensile/transverse stress perpendicular to the load direction), which goes along with a variation of the associated displacement fields. We may speculate that such a deformation state may give rise to a specific polar angular dependence in the neutron-scattering signal.

The hard magnetic samples with the composition $\left(\mathrm{Nd}_{60} \mathrm{Fe}_{30} \mathrm{Al}_{10}\right)_{92} \mathrm{Ni}_{8}$ (nominal atomic percent) were produced at Universität des Saarlandes by means of suction casting. Master ingots of the alloy were produced from high-purity elements $(99.9 \%)$ in an electric arc melter under high-purity Ar atmosphere. Pieces of the master ingots were then remelted in a customized suction casting arc-melting device under a high-purity atmosphere and cast into a water-cooled copper mold, yielding thin plates with dimensions of approximately $20 \times 10 \times 0.6 \mathrm{~mm}$. Three disks with thicknesses of, respectively, $0.48,0.63$, and $0.52 \mathrm{~mm}$ were wire-cut from the 
ground plates. One sample remained in the as-cast state, one specimen was aged (annealed at $300{ }^{\circ} \mathrm{C}$ for $10 \mathrm{~h}$ in $\mathrm{N}_{2}$ atmosphere), and one was first aged (annealed at $300^{\circ} \mathrm{C}$ in $\mathrm{N}_{2}$ atmosphere for $10 \mathrm{~h}$ ) and then mechanically deformed. The specimen was aged before deformation to relax the sample and thereby approach the metastable ground state. The rationale behind this treatment is the following: it seemed plausible to conjecture when deforming the hard magnetic material (with the comparatively strong magnetoelastic coupling) by starting out from the near-metastable ground state, since we then may obtain a maximal signal due to spin reorientation decorating the emerging displacement field. The deformation force was applied in the direction normal to the plane of the disk, with a resulting reduction of disk thickness from 0.52 to $0.51 \mathrm{~mm}$. In contrast to the deformation mode of the soft magnetic material, the hard magnets suffered a buckling instability when load was applied in-plane. Inducing stress normal to the plane of the disk results in a homogeneous compressive stress state across the disk, and hence it is common to characterize the resulting strain as overall true plastic strain, which amounts to $\approx 2.1 \%$.

The amorphous character of all the samples was confirmed before and after the heat or mechanical-deformation treatments by means of x-ray wide-angle diffraction and transmission electron microscopy. The magnetic properties were studied using a $14 \mathrm{~T}$ vibrating sample magnetometer (Cryogenic VSM). Magnetization data were recorded at room temperature; the applied-field direction was in the plane of the samples (as in the SANS experiments).

\section{THEORY}

\section{A. Magnetic SANS}

When the applied magnetic field $\mathbf{H}_{0}=H_{0} \mathbf{e}_{z}$ is perpendicular to the wave vector $\mathbf{k}_{0}=k_{0} \mathbf{e}_{x}$ of the incoming neutron beam (see Fig. 1), the unpolarized total elastic SANS cross section $d \Sigma / d \Omega$ can be written as [12]

$$
\begin{aligned}
\frac{d \Sigma}{d \Omega}(\mathbf{q})= & \frac{8 \pi^{3}}{V} b_{H}^{2}\left(\frac{|\tilde{N}|^{2}}{b_{H}^{2}}+\left|\tilde{M}_{x}\right|^{2}+\left|\tilde{M}_{y}\right|^{2} \cos ^{2} \theta\right. \\
& \left.+\left|\tilde{M}_{z}\right|^{2} \sin ^{2} \theta-\left(\tilde{M}_{y} \tilde{M}_{z}^{*}+\tilde{M}_{y}^{*} \tilde{M}_{z}\right) \sin \theta \cos \theta\right),
\end{aligned}
$$

where $\mathbf{q}$ denotes the scattering or momentum-transfer vector ( $q=4 \pi \lambda^{-1} \sin \psi$ ), $V$ is the scattering volume, the constant $b_{H}=2.91 \times 10^{8} \mathrm{~A}^{-1} \mathrm{~m}^{-1}$ relates the atomic magnetic moment to the Bohr magneton, $\widetilde{N}(\mathbf{q})$ and $\tilde{\mathbf{M}}(\mathbf{q})=\left\{\tilde{M}_{x}, \widetilde{M}_{y}, \widetilde{M}_{z}\right\}$ denote, respectively, the Fourier transforms of the nuclear scattering-length density and the magnetization vector field $\mathbf{M}(\mathbf{r})=\left\{M_{x}, M_{y}, M_{z}\right\}, \theta$ is the angle between $\mathbf{H}_{0}$ and $\mathbf{q}$ so that $\mathbf{q} \cong q\{0, \sin \theta, \cos \theta\}$ in the small-angle approximation, and the asterisks mark the complex-conjugate quantity. Note that for this scattering geometry, Fourier components with $q_{x} \cong 0$ are probed.

At a saturating field, the magnetization is given by $\mathbf{M}(\mathbf{r})=$ $\left\{0,0, M_{z}(\mathbf{r})=M_{s}(\mathbf{r})\right\}$, where $M_{s}(\mathbf{r})$ describes the spatial variation of the local saturation magnetization. In this situation, $d \Sigma / d \Omega$ reduces to the so-called residual scattering cross section

$$
\frac{d \Sigma_{\mathrm{res}}}{d \Omega}(\mathbf{q})=\frac{8 \pi^{3}}{V}\left(|\tilde{N}|^{2}+b_{H}^{2}\left|\tilde{M}_{s}\right|^{2} \sin ^{2} \theta\right),
$$

where $\widetilde{M}_{s}(\mathbf{q})$ is the Fourier transform of $M_{s}(\mathbf{r})$. For magnetic fields below saturation, one may express the total cross section $d \Sigma / d \Omega$ as

$$
\frac{d \Sigma}{d \Omega}(\mathbf{q})=\frac{d \Sigma_{\mathrm{res}}}{d \Omega}(\mathbf{q})+\frac{d \Sigma_{M}}{d \Omega}(\mathbf{q})
$$

where

$$
\begin{aligned}
\frac{d \Sigma_{M}}{d \Omega}(\mathbf{q})= & \frac{8 \pi^{3}}{V} b_{H}^{2}\left[\left|\tilde{M}_{x}\right|^{2}+\left|\tilde{M}_{y}\right|^{2} \cos ^{2} \theta+\left(\left|\tilde{M}_{z}\right|^{2}-\left|\tilde{M}_{s}\right|^{2}\right)\right. \\
& \left.\times \sin ^{2} \theta-\left(\tilde{M}_{y} \tilde{M}_{z}^{*}+\tilde{M}_{y}^{*} \tilde{M}_{z}\right) \sin \theta \cos \theta\right]
\end{aligned}
$$

denotes the so-called spin-misalignment SANS cross section. Note that $\widetilde{M}_{z} \cong \widetilde{M}_{s}$ in the approach-to-saturation regime, so that in this case $d \Sigma_{M} / d \Omega$ reduces to

$$
\begin{aligned}
\frac{d \Sigma_{M}}{d \Omega}(\mathbf{q})= & \frac{8 \pi^{3}}{V} b_{H}^{2}\left(\left|\tilde{M}_{x}\right|^{2}+\left|\tilde{M}_{y}\right|^{2} \cos ^{2} \theta\right. \\
& \left.-\left(\tilde{M}_{y} \tilde{M}_{z}^{*}+\tilde{M}_{y}^{*} \tilde{M}_{z}\right) \sin \theta \cos \theta\right) .
\end{aligned}
$$

$d \Sigma_{M} / d \Omega$ contains the purely magnetic scattering due to the transversal spin components, with related Fourier amplitudes $\widetilde{M}_{x}(\mathbf{q})$ and $\widetilde{M}_{y}(\mathbf{q})$. Note that $d \Sigma_{M} / d \Omega$, which vanishes at complete saturation, also depends on spatial variations of the magnetization via the longitudinal Fourier component $\widetilde{M}_{z}$ in the $\widetilde{M}_{y}-\widetilde{M}_{z}$ cross term.

Further analysis of the magnetic SANS cross section requires expressions for the magnetization Fourier amplitudes, which depend on the particular model for the magnetic material. In Refs. [18,19], a theory of magnetic SANS based on the continuum theory of micromagnetics has been developed. The approach considers two origins of spin misalignment: (i) spatial nanometer-scale variations in the orientation and magnitude of the magnetic anisotropy field $\mathbf{H}_{p}(\mathbf{r})$, and (ii) spatial nanometer-scale variations of the saturation magnetization $M_{s}(\mathbf{r})$. This scenario appears to be well adapted to the inhomogeneous magnetic microstructure, which is expected to be present in BMGs. Within this framework, $d \Sigma_{M} / d \Omega$ can be decomposed into two contributions, which are related to the above-mentioned sources of spin misalignment, a contribution $S_{H} R_{H}$ due to perturbing magnetic anisotropy fields, and a contribution $S_{M} R_{M}$ due to magnetostatic fields:

$$
\frac{d \Sigma_{M}}{d \Omega}(\mathbf{q})=S_{H}(\mathbf{q}) R_{H}\left(q, \theta, H_{i}\right)+S_{M}(\mathbf{q}) R_{M}\left(q, \theta, H_{i}\right),
$$

where the anisotropy-field scattering function

$$
S_{H}(\mathbf{q})=\frac{8 \pi^{3}}{V} b_{H}^{2}|h|^{2}
$$

depends on the Fourier coefficient $\mathbf{h}(\mathbf{q})$ of the magnetic anisotropy field $\mathbf{H}_{p}(\mathbf{r})$, whereas the scattering function of the longitudinal magnetization

$$
S_{M}(\mathbf{q})=\frac{8 \pi^{3}}{V} b_{H}^{2}\left|\tilde{M}_{z}\right|^{2}
$$


depends on the Fourier coefficient $\tilde{M}_{z}(\mathbf{q})$ of the magnetization component $M_{z}(\mathbf{r})$. The corresponding micromagnetic response functions are dimensionless:

$$
R_{H}\left(q, \theta, H_{i}\right)=\frac{p^{2}}{2}\left(1+\frac{\cos ^{2} \theta}{\left(1+p \sin ^{2} \theta\right)^{2}}\right)
$$

and

$$
R_{M}\left(q, \theta, H_{i}\right)=\frac{p^{2} \sin ^{2} \theta \cos ^{4} \theta}{\left(1+p \sin ^{2} \theta\right)^{2}}+\frac{2 p \sin ^{2} \theta \cos ^{2} \theta}{1+p \sin ^{2} \theta},
$$

where the dimensionless function

$$
p\left(q, H_{i}\right)=\frac{M_{s}}{H_{i}\left(1+l_{H}^{2} q^{2}\right)}
$$

depends on the internal magnetic field

$$
H_{i}=H_{0}-N M_{S}
$$

and on the exchange length

$$
l_{H}\left(H_{i}\right)=\sqrt{\frac{2 A}{\mu_{0} M_{s} H_{i}}} .
$$

The function $l_{H}\left(H_{i}\right)$ specifies the range over which perturbations in the microstructure in the vicinity of defects are transmitted by the exchange interaction into the surroundings ( $M_{s}$ is the macroscopic saturation magnetization of the sample, $A$ is the exchange-stiffness parameter, $N$ is the demagnetizing factor, and $\mu_{0}=4 \pi \times 10^{-7} \mathrm{~T} \mathrm{~m} / \mathrm{A}$ ).

We note that for a statistically isotropic material, the functions $S_{H}$ and $S_{M}$ depend only on the magnitude $q$ of the scattering vector $\mathbf{q}$, not on its orientation. Moreover, since $S_{H}(q)$ and $S_{M}(q)$ are, respectively, related to the strength and spatial structures of the magnetic anisotropy field and magnetostatic field, it is clear that the corresponding ratio decisively determines the properties of $d \Sigma_{M} / d \Omega$ such as the angular anisotropy (compare, e.g., the expressions for $R_{H}$ and $R_{M}$ ), the asymptotic power-law exponent, and the characteristic decay length of spin-misalignment fluctuations [18].

Since the continuum theory of micromagnetics is also applicable to amorphous ferromagnets [20], the predictions of the micromagnetic SANS theory are expected to be transferable to the magnetic BMGs that are studied in this paper. However, an important difference between conventional (nano)crystalline ferromagnets and magnetic BMGs relates to the characteristic length scales over which perturbations in the magnetic anisotropy field and in the saturation magnetization manifest: while in nanocrystalline systems $\mathbf{H}_{p}$ and $M_{s}$ typically vary on a scale of the grain size, say, $10 \mathrm{~nm}$, in magnetic BMGs this length scale is defined by the interatomic distance due to atomic-site anisotropy and randomness. As a consequence, the functions $S_{H}(q)$ and $S_{M}(q)$ are almost constant for magnetic BMGs (i.e., $q$-independent) over a large part of the experimentally accessible range of momentum transfers $\left(0.01 \lesssim q \lesssim 1.0 \mathrm{~nm}^{-1}\right)$. Therefore, the asymptotic power-law dependence of the spin-misalignment scattering cross section $d \Sigma_{M} / d \Omega=S_{H} R_{H}+S_{M} R_{M}$ is determined by the asymptotic behavior of both response functions, $R_{H} \propto q^{-4}$ and $R_{M} \propto q^{-2}$, in other words, $d \Sigma_{M} / d \Omega \propto q^{-n}$ with $2 \leqslant n \leqslant 4$. The results for the field-dependent exponent $n$, obtained on the total $d \Sigma / d \Omega$ of the rare-earth containing BMGs, seem to confirm this prediction (see Fig. 3 in the Supplemental Material [13]).

With reference to the fourfold angular anisotropy of experimentally observed spatial strain autocorrelations [8], it becomes clear by inspection of the response function $R_{M}$ [Eq. (10)] that clover-leaf-type anisotropies (with maxima roughly along the detector diagonals) can already result from spatial variations in $M_{s}$; in other words, magnetic SANS may not be able to unambiguously separate scattering contributions due to $M_{s}(\mathbf{r})$ and due to displacement-field-induced spin textures.

\section{B. Correlation function of the spin-misalignment SANS cross section}

Since SANS measurements yield scattering data in momentum space, obtaining real-space characteristics of the material requires some additional analysis. One of the most widely implemented procedures in small-angle scattering is the correlation-function analysis [21-24]. Here, we define the correlation function $c(\mathbf{r})$ of the spin-misalignment SANS cross section as the Fourier transform of $d \Sigma_{M} / d \Omega$ [25]. In a SANS experiment, only the components of the momentum-transfer vector $\mathbf{q}$ perpendicular to the incident-beam direction $\mathbf{k}_{0}$ are effectively probed. For $\mathbf{k}_{0} \perp \mathbf{H}_{0}\left(q_{x} \cong 0\right)$, this implies that $\frac{d \Sigma_{M}}{d \Omega} \cong \frac{d \Sigma_{M}}{d \Omega}\left(q_{y}, q_{z}\right)$, and the normalized correlation function $c(y, z)$ is obtained as

$$
c(y, z)=\frac{\int_{-\infty}^{+\infty} \int_{-\infty}^{+\infty} \frac{d \Sigma_{M}}{d \Omega}\left(q_{y}, q_{z}\right) \cos \left(q_{y} y+q_{z} z\right) d q_{y} d q_{z}}{\int_{-\infty}^{+\infty} \int_{-\infty}^{+\infty} \frac{d \Sigma_{M}}{d \Omega}\left(q_{y}, q_{z}\right) d q_{y} d q_{z}} .
$$

Equation (14) can be reduced to one dimension by performing an angular averaging over all directions in the detector plane. As shown in Ref. [25], this leads to

$$
c(r)=\frac{\int_{0}^{\infty} \frac{d \Sigma_{M}}{d \Omega}(q) J_{0}(q r) q d q}{\int_{0}^{\infty} \frac{d \Sigma_{M}}{d \Omega}(q) q d q},
$$

where $J_{0}(x)$ denotes the Bessel function of zeroth order.

Generally, for magnetic neutron scattering, both $\frac{d \Sigma_{M}}{d \Omega}\left(q_{y}, q_{z}\right)$ and $c(y, z)$ are anisotropic functions, so that the value of the correlation length that is determined from $c(y, z)$ also depends on the orientation. To extract a single average correlation length $l_{C}$ (of the spin misalignment) from the data, we have employed the averaged correlation function, Eq. (15); as a criterion, we used the $\exp (-1)$ decay length, i.e., $c\left(r=l_{C}\right)=$ $\exp (-1)$. This value represents a measure for the size of inhomogeneously magnetized regions around microstructural defects $[25,26]$. Note that the above definition of $l_{C}$ as the $\exp (-1)$ length does not imply that the correlation function decays exponentially, it is merely an operational criterion.

In our SANS experiments, the range of scattering vectors was limited to $0.035 \lesssim q \lesssim 1.5 \mathrm{~nm}^{-1}$. It is a well-known result of the theory of Fourier transformation that the maximum value of the momentum transfer $q_{\max }$ limits the resolution in real space to roughly $\Delta r \gtrsim \pi / q_{\max } \cong 2 \mathrm{~nm}$ and that the size of the largest structure in the system is given by $r_{\max } \cong \pi / q_{\min } \cong$ $90 \mathrm{~nm}$ [27]. The correlation function $c(r)$, Eq. (15), was computed by means of direct Fourier transformation within 
$r_{\min }=2 \pi / q_{\max } \cong 4 \mathrm{~nm}$ and $r_{\max }$. For the numerical computation of $c(r)$, the integrals in Eq. (15) were approximated by discrete sums; in order to reduce termination effects, the experimental spin-misalignment scattering data beyond $q_{\max }$ were extrapolated to infinity using power laws of the form $d \Sigma_{M} / d \Omega \propto q^{-n}$ with $n \geqslant 4$, and the extrapolation from $q_{\min }$ to $q=0$ was carried out using different schemes (constant, linear, Guiner law). We emphasize that the total nuclear and magnetic SANS data at the highest experimental magnetic fields (1.5 $\mathrm{T}$ for the soft magnetic BMG and $8 \mathrm{~T}$ for the hard magnetic BMG) were subtracted from the measurements at lower fields; this implies that $d \Sigma_{M} / d \Omega$ does not contain the coherent and incoherent nuclear SANS.

For the computation of the correlation function, one may also use Glatter's indirect Fourier transformation method [24,27-31]. However, this technique, which was originally developed for particle scattering, requires the rather precise knowledge of the maximum particle size in the system. Since the magnetic SANS that is investigated in this study has its origin in continuously varying magnetic-field-dependent magnetization profiles (no sharp boundaries), we have employed the direct Fourier transformation technique for obtaining rough information on the characteristic real-space length scales.

\section{RESULTS AND DISCUSSION}

\section{A. $\mathrm{Fe}_{70} \mathrm{Mo}_{5} \mathrm{Ni}_{5} \mathrm{P}_{12.5} \mathrm{~B}_{2.5} \mathrm{C}_{5}$}

The results of the magnetization measurements are shown in Fig. 2. All samples are classified as soft magnetic, and a field of the order of $0.2 \mathrm{~T}$ is sufficient to magnetically saturate them. The saturation magnetization of the aged sample $\left(M_{s}=111.6 \mathrm{emu} \mathrm{g}^{-1}\right)$ is slightly larger than the saturation magnetization of the other two samples $\left(M_{s}=109.5 \mathrm{emu} \mathrm{g}^{-1}\right.$ for the as-cast and $M_{s}=108.3 \mathrm{emu} \mathrm{g}^{-1}$ for the deformed

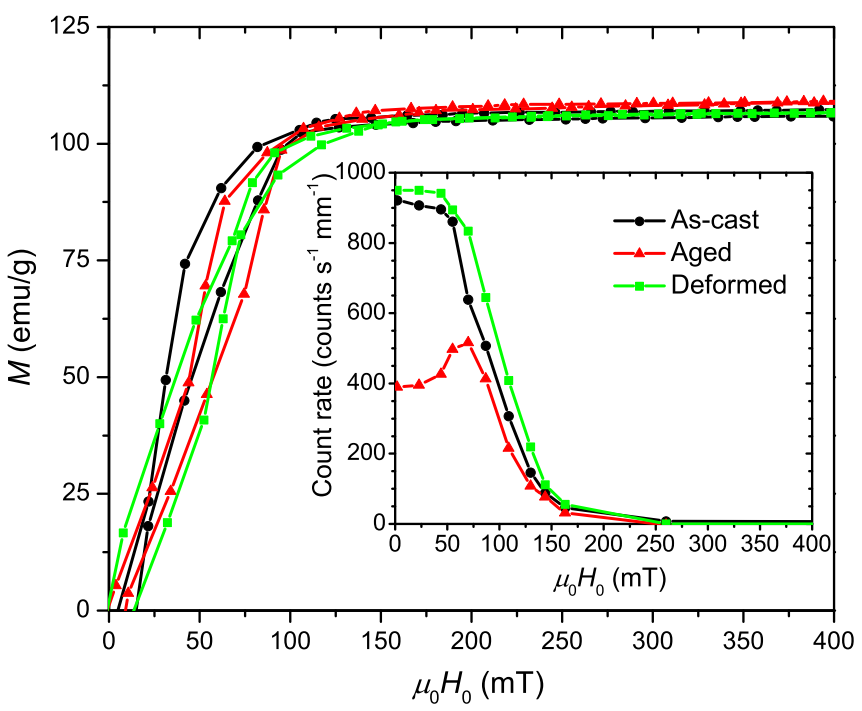

FIG. 2. Room-temperature magnetization curves of as-cast, aged, and deformed $\mathrm{Fe}_{70} \mathrm{Mo}_{5} \mathrm{Ni}_{5} \mathrm{P}_{12.5} \mathrm{~B}_{2.5} \mathrm{C}_{5}$ (only the upper right quadrant is shown). The inset shows the field dependence of the neutron count rate of the samples normalized by the respective sample thickness. The measured $q$-range for the count rate is $0.035 \lesssim q \lesssim 0.3 \mathrm{~nm}^{-1}$.
BMG). The inset in Fig. 2 depicts the field dependence of the total detector count rate. In the saturated state, the count rate is extremely low (only $1-2$ counts $\mathrm{s}^{-1} \mathrm{~mm}^{-1}$ ), which indicates a quite weak residual scattering cross section [compare Eqs. (1) and (2)]. Reducing the applied field below $200 \mathrm{mT}$ results in a strong increase of the count rate [compare with the $M\left(H_{0}\right)$ data in Fig. 2], which suggests that the measured total $d \Sigma / d \Omega$ is dominated by the spin-misalignment scattering [compare Eqs. (1)-(4)]. The as-cast and deformed samples assume at zero and low fields a plateau value of the count rate to then monotonically decrease and practically vanish beyond $250 \mathrm{mT}$; the aged sample starts out from a much lower count rate at zero field to then increase and assume a maximum at around $80 \mathrm{mT}$, followed by a monotonic decrease similar to the other samples. This difference in count-rate behavior between the samples is, however, not reflected in the $M\left(H_{0}\right)$ data. Note that for fields below about $50 \mathrm{mT}$, the count rates of all samples change only very weakly while the values of $M\left(H_{0}\right)$ keep decreasing. Currently, we have no clear understanding of the maximum in the count rate of the aged sample; below, we will see that this feature is accompanied by the observation of an unusual angular anisotropy in the two-dimensional SANS pattern, so that it might be related to the formation of a domain structure.

The experimental two-dimensional unpolarized SANS cross sections $d \Sigma / d \Omega$ are displayed in Fig. 3. In agreement with the field dependence of the count rates, the scattering at saturation (left column in Fig. 3, $\mu_{0} H_{0}=1496 \mathrm{mT}$ ) is much weaker for all samples than the scattering at lower fields; additionally, $d \Sigma / d \Omega$ at saturation is isotropic. From the expression for the residual SANS cross section [Eq. (2)], one may see that $d \Sigma_{\text {res }} / d \Omega$ consists of two terms: the isotropic nuclear scattering $\sim|\widetilde{N}|^{2}$, and the anisotropic longitudinal magnetic scattering $\sim\left|\widetilde{M}_{z}\right|^{2} \sin ^{2} \theta$. Note that for statistically isotropic samples, both Fourier components $|\widetilde{N}|^{2}$ and $\left|\widetilde{M}_{z}\right|^{2}$ are isotropic [12]. Therefore, the observed isotropic pattern of the residual scattering cross section strongly indicates that the nuclear scattering term dominates $d \Sigma_{\text {res }} / d \Omega$, i.e., $|\widetilde{N}|^{2} \gg$ $b_{H}^{2}\left|\widetilde{M}_{z}\right|^{2}$. Moreover, since $d \Sigma_{\text {res }} / d \Omega$ and, hence, $|\widetilde{N}|^{2}$ is much smaller than the spin-misalignment SANS cross section (compare also Fig. 5 in the Supplemental Material [13]), this then implies that $d \Sigma_{M} / d \Omega=S_{H} R_{H}+S_{M} R_{M}$ is dominated by the anisotropy-field-related scattering term $S_{H} R_{H}\left(S_{H} \propto\right.$ $|h|^{2}$ ), rather than by the magnetostatic contribution $S_{M} R_{M}$ $\left(S_{M} \propto\left|\tilde{M}_{z}\right|^{2}\right)$ [compare Eqs. (6)-(8)].

With decreasing field down to the remanent state, the two-dimensional SANS patterns in Fig. 3 reveal a combination of different angular anisotropies, which can be related to the different magnetization Fourier components in Eq. (1). Overall, the cross sections in Fig. 3 exhibit (for fields down to a few $10 \mathrm{mT}$ ) an elongation in the direction of the horizontal field direction, which is a strong indication for the presence of long-range spin misalignment [cf. the term $\left|\widetilde{M}_{y}\right|^{2} \cos ^{2} \theta$ in Eq. (1)]. In particular, we refer to the data at $70 \mathrm{mT}$, which show (at small $q$ ) a pronounced sharp maximum of the neutron intensity for directions parallel and antiparallel to the applied field. This so-called "spike anisotropy" has previously been observed in a SANS study on sintered Nd-Fe-B magnets [32], and it is related to the formation of flux-closure patterns on a nanometer length scale; more specifically, it is related to 

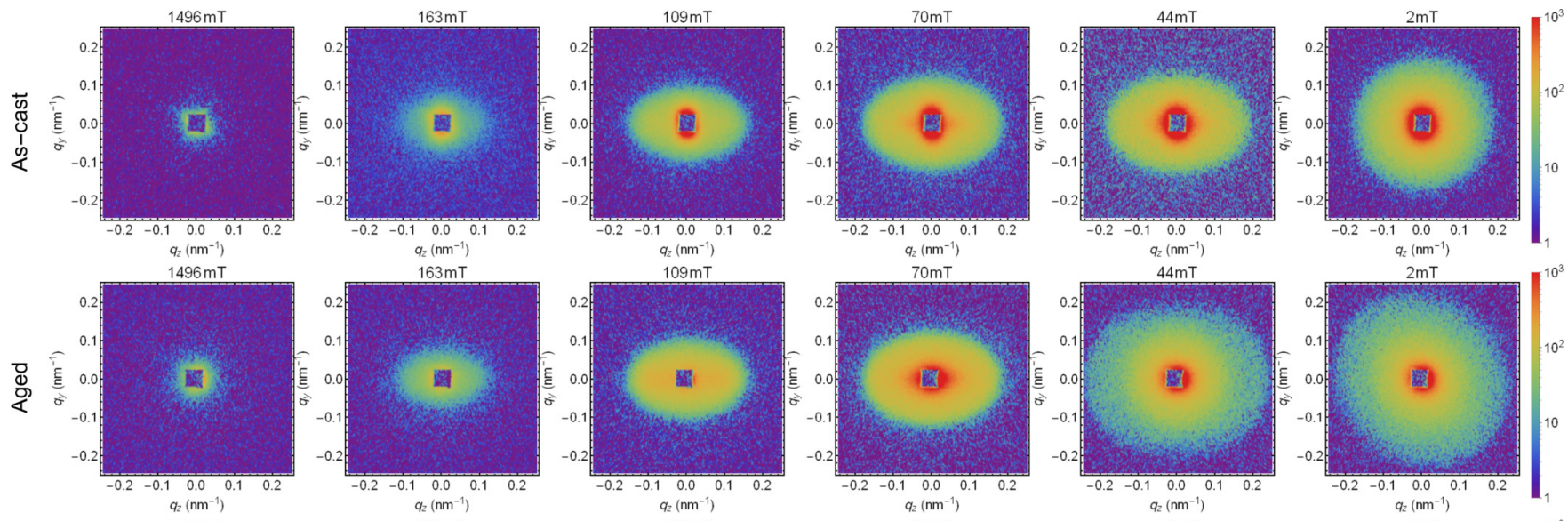

$1496 \mathrm{mT}$
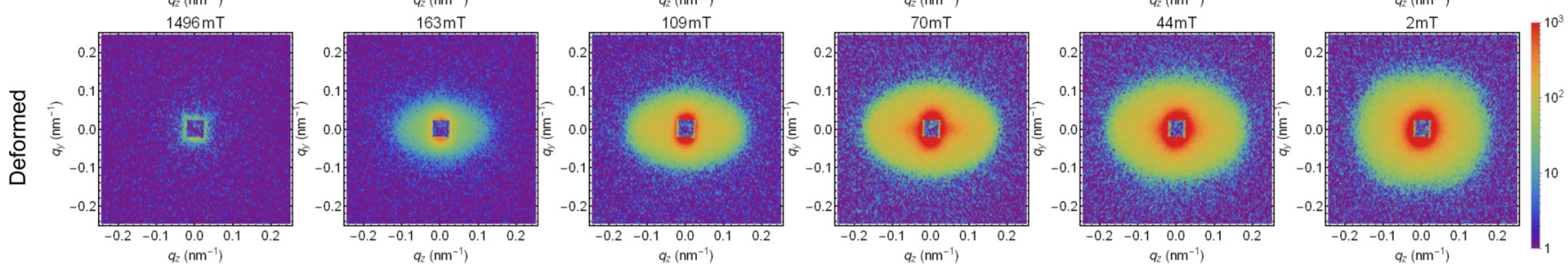

FIG. 3. Two-dimensional total (nuclear and magnetic) unpolarized SANS cross sections $d \Sigma / d \Omega$ of as-cast, aged, and deformed BMG alloy $\mathrm{Fe}_{70} \mathrm{Mo}_{5} \mathrm{Ni}_{5} \mathrm{P}_{12.5} \mathrm{~B}_{2.5} \mathrm{C}_{5}$ at selected applied magnetic fields (logarithmic color scale). $\mathbf{H}_{0}$ is horizontal in the plane. Note the relatively pronounced and sharp maximum ("spike") of the $d \Sigma / d \Omega$ of the deformed sample at $70 \mathrm{mT}$ and low $q$.

the term $p \sin ^{2} \theta$ in the denominator of the response function $R_{H}$ [Eq. (9)]. Figure 4 represents the angular dependence of $d \Sigma_{M} / d \Omega$ taken at $\mu_{0} H_{0}=70 \mathrm{mT}$ for a set of $q$ values near the beamstop (red refers to small $q$ ) and near the borders of the detector (dark blue refers to large $q$ ). While the aged sample shows maxima only at angles $\theta \sim 0^{\circ}$ and $180^{\circ}$, which are related to a horizontal elongation of $d \Sigma / d \Omega$, the as-cast and deformed samples both exhibit additional peaks at $\theta \sim 90^{\circ}$, which quickly fade away with increasing $q$. This behavior can best be observed at fields of 109 and $70 \mathrm{mT}$, although it is present down to the remanent state. When approaching zero field, the SANS cross sections tend to be more isotropic, although for the aged sample $d \Sigma / d \Omega$ reveals an elongated angular anisotropy oriented at about $135^{\circ}$ relative to the horizontal direction. This feature might be related to the unusual low-field behavior of the count rate in Fig. 2; to this end, we can only speculate that its origin may be related to the formation of a domain structure absent in the other two specimens. No presence of a clover-leaf-shaped angular anisotropy (with maxima roughly along the detector diagonals, cf. the angular variation of $R_{M}$ ) can be found in Fig. 3 . As discussed before, this can be related to the fact that the spin-misalignment SANS cross section of the soft magnetic BMG is dominated by the term $S_{H} R_{H}$ due to the magnetic anisotropy field.

The radially averaged spin-misalignment scattering cross sections were obtained by integration of the corresponding two-dimensional data over an angular range of $2 \pi$, and they are displayed in Fig. 5. In agreement with the count rate in Fig. 2, all samples show strong spin-misalignment scattering with a change of $d \Sigma_{M} / d \Omega$ by up to two orders of magnitude between saturation and $0 \mathrm{~T}$. For the as-cast and deformed samples, the scattering increases monotonously with decreasing field down to $\sim 70 \mathrm{mT}$, and then it remains
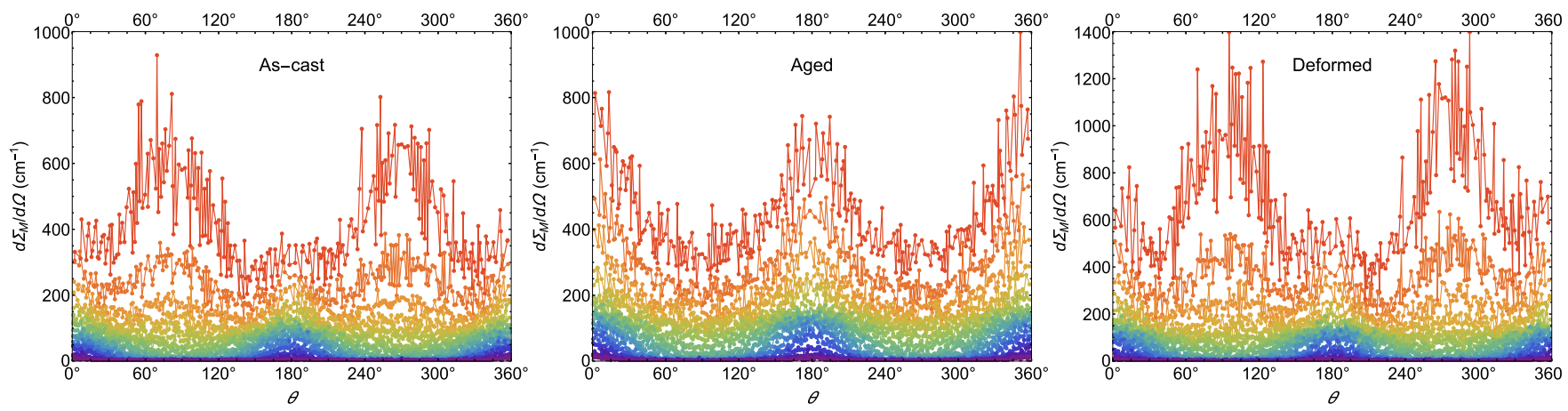

FIG. 4. Comparison of the angular $\theta$-dependence of $d \Sigma_{M} / d \Omega$ of as-cast, aged, and deformed $\mathrm{Fe}_{70} \mathrm{Mo}_{5} \mathrm{Ni}_{5} \mathrm{P}_{12.5} \mathrm{~B}_{2.5} \mathrm{C}_{5}$ at $\mu_{0} H_{0}=70 \mathrm{mT}$. Red corresponds to low $q$-values around the beamstop $\left(q \cong 0.035 \mathrm{~nm}^{-1}\right)$, while blue represents high $q$-values from around the borders of the detector $\left(q \cong 0.2 \mathrm{~nm}^{-1}\right)$. 

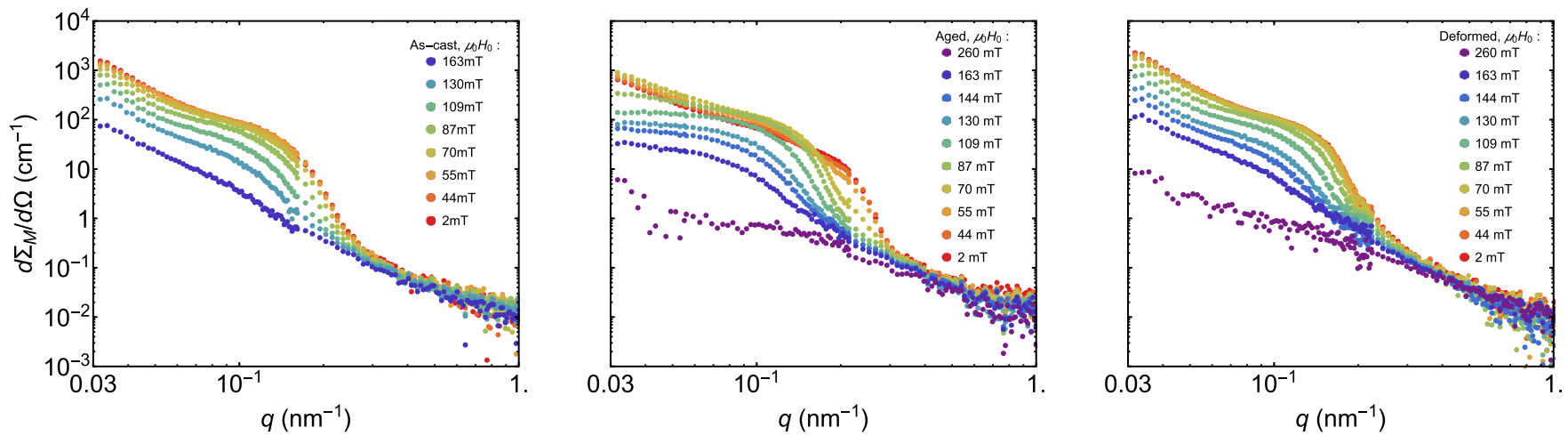

FIG. 5. Azimuthally averaged spin-misalignment SANS cross sections $d \Sigma_{M} / d \Omega$ of as-cast, aged, and deformed $\mathrm{Fe}_{70} \mathrm{Mo}_{5} \mathrm{Ni}_{5} \mathrm{P}_{12.5} \mathrm{~B}_{2.5} \mathrm{C}_{5}$ at selected applied magnetic fields (see the insets, log-log scale). The respective scattering at saturation (1496 mT) has been subtracted in each data set.

essentially unchanged for smaller fields; note, however, that (for these samples) the angular anisotropy of the cross sections in Fig. 3 continues to change even for $\mu_{0} H_{0}<70 \mathrm{mT}$. For the aged sample, $d \Sigma_{M} / d \Omega$ increases with decreasing field down to $\sim 55 \mathrm{mT}$, and then it begins decreasing with a concomitant shifting of the shoulder in the SANS curves to larger $q$-values. Spin-misalignment scattering on a scale above $q \gtrsim 0.5 \mathrm{~nm}^{-1}$ becomes essentially field-independent for all samples. The comparison of $d \Sigma_{M} / d \Omega$ for all three samples is shown in Fig. 6. The as-cast and deformed samples show a qualitatively similar behavior, with the deformed sample exhibiting a larger spin-misalignment scattering. It also becomes visible in Figs. 5 and 6 that with increasing field, the shoulders in $d \Sigma_{M} / d \Omega$ evolve to smaller $q$-values and basically vanish or disappear from the accessible range of momentum transfers.

The difference between the aged sample and the as-cast and deformed samples becomes more evident when inspecting the two-dimensional correlation functions $c(y, z)$ (Fig. 7). Since the spin-misalignment SANS cross sections are highly anisotropic, this feature also transfers to the correlation functions. The $c(y, z)$ of the aged sample for fields above $44 \mathrm{mT}$ are strongly elongated along the vertical direction (which is

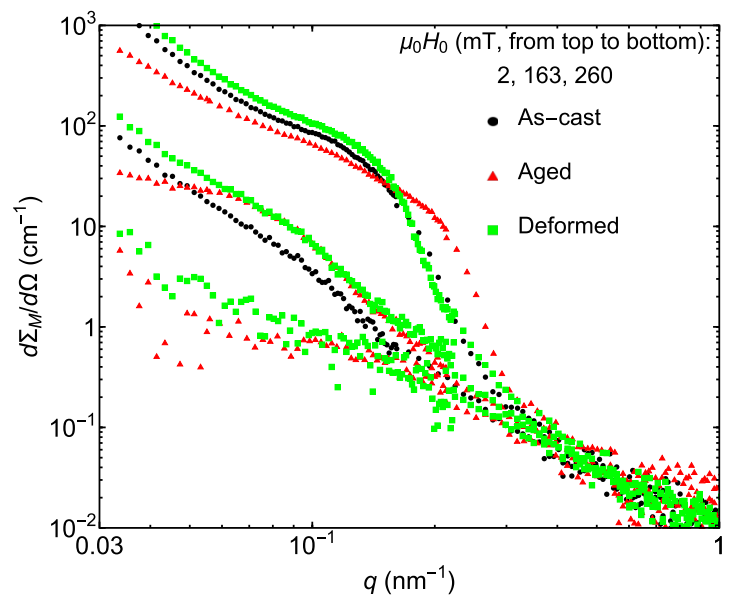

FIG. 6. Comparison of $d \Sigma_{M} / d \Omega$ of as-cast, aged, and deformed $\mathrm{Fe}_{70} \mathrm{Mo}_{5} \mathrm{Ni}_{5} \mathrm{P}_{12.5} \mathrm{~B}_{2.5} \mathrm{C}_{5}$ at selected applied magnetic fields (see the insets, log-log scale). perpendicular to the field direction). At $109 \mathrm{mT}$, the $c(y, z)$ of the aged sample exhibits negative values for $20 \lesssim r \lesssim 50 \mathrm{~nm}$ (shown in dark purple), except for the values in the direction perpendicular to the field. In nuclear SANS, negative values of the distance distribution function $p(r)=r^{2} \gamma(r)$ are attributed to distances that connect regions with opposite sign of the scattering length density more frequently than regions with the same sign [27]. However, for magnetic SANS, such a straightforward interpretation of the correlation function of the spin-misalignment SANS cross section in terms of a specific magnetization distribution cannot be made. This is because $c(y, z)$ - being the Fourier transform of $d \Sigma_{M} / d \Omega$ [Eq. (5)]-is not the autocorrelation function of the magnetization, in contrast to $\gamma(r)$, which is the autocorrelation function of the nuclear density $N(\mathbf{r})$. Nevertheless, the observation that $c(y, z)$ of the aged sample is negative at $109 \mathrm{mT}$ and for $20 \lesssim r \lesssim 50 \mathrm{~nm}$ may indicate the existence of magnetization components that are oriented opposite to a given magnetic moment at the origin.

For the as-cast and deformed samples, the $c(y, z)$ show (at large $r$ ) an increase of the horizontal component with decreasing field, in agreement with the two-dimensional scattering data in Fig. 3, which reveal an increasing vertical component at small $q$. Likewise, for small values of $r$, corresponding to large $c$-values $(c \gtrsim 0.8)$, the anisotropy of the correlation function shows a maximum along the vertical direction for all samples, corresponding to a horizontal elongation in $\mathbf{q}$ space. Moreover, the $c(y, z)$ of the aged sample decay faster than the $c(y, z)$ of the other two samples.

To extract a single average correlation length from the data, we have computed the field-dependent $2 \pi$-averaged SANS cross sections, and we have estimated the correlation length $l_{C}$ as the $\exp (-1)$ length from the numerically computed correlation functions according to Eq. (15). As an example, Fig. 11 depicts such correlation functions $c(r)$ of the rareearth-based BMGs, which we are going to discuss in the next paragraph. The results for $l_{C}\left(H_{0}\right)$ of the soft magnetic BMGs are shown in Fig. 8. The correlation lengths of the as-cast and deformed samples vary from about 5 to $25 \mathrm{~nm}$, while the $l_{C}$ of the aged sample takes on values of $\sim 5-20 \mathrm{~nm}$ (resembling the count rate behavior in Fig. 2). Regarding the absolute $l_{C}$ values, one recognizes that the aged BMG exhibits the smallest correlation lengths; this can be explained 

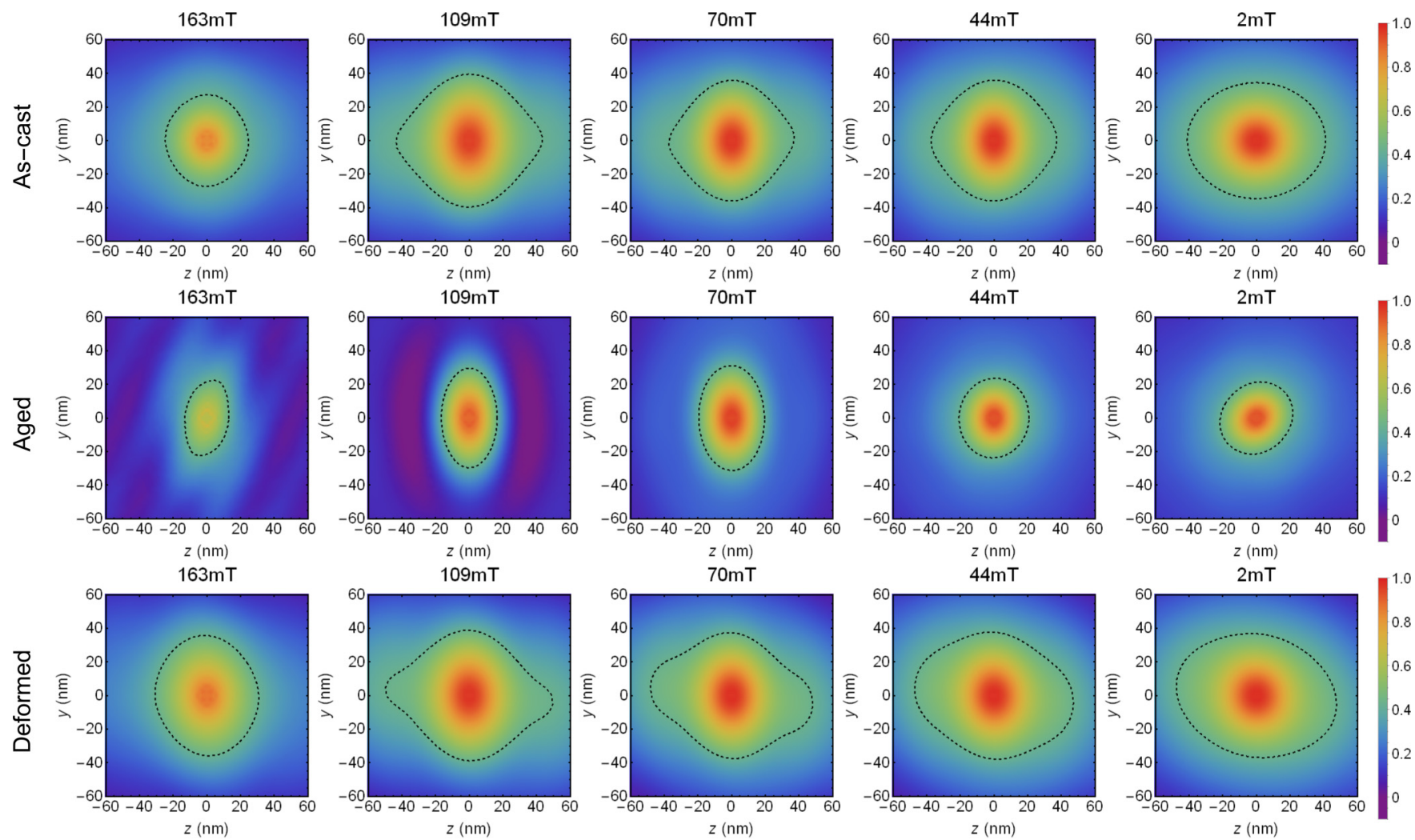

FIG. 7. Field dependence of the two-dimensional (normalized) correlation functions $c(y, z)$ [computed according to Eq. (14)] of as-cast, aged, and deformed $\mathrm{Fe}_{70} \mathrm{Mo}_{5} \mathrm{Ni}_{5} \mathrm{P}_{12.5} \mathrm{~B}_{2.5} \mathrm{C}_{5}$ at selected applied magnetic fields (see the insets). $\mathbf{H}_{0}$ is horizontal in the plane. Dashed contour lines: $c(y, z)=\exp (-1)$.

by the fact that the aging procedure $\left(8 \mathrm{~h}\right.$ at $\left.358^{\circ} \mathrm{C}\right)$ goes along with enthalpy and concomitant structural relaxation, which then results in a smaller size of nonuniform magnetization structures. In agreement with this assessment, we see in the magnetization data (Fig. 2) that the aged sample exhibits a slightly larger high-field magnetization than the other two samples; on the other hand, the deformed sample has the smallest magnetization and the largest $l_{C}$ values. The results

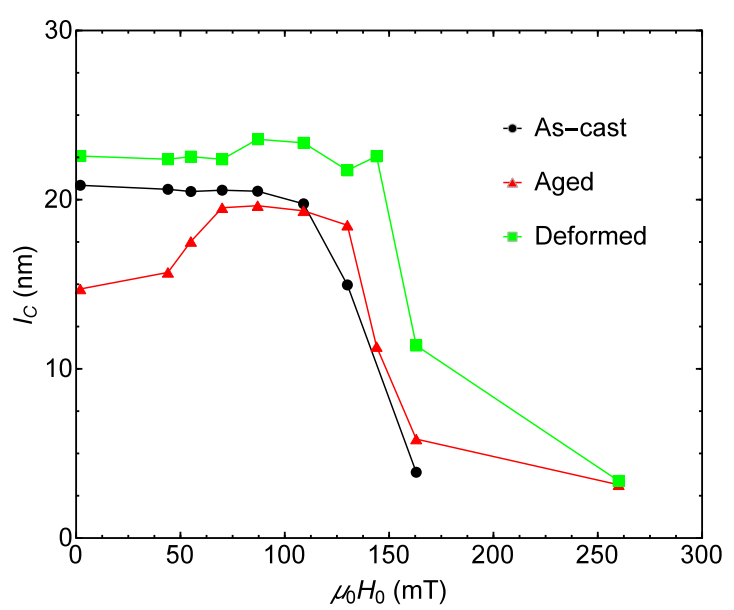

FIG. 8. Field dependence of the correlation length $l_{C}$ of as-cast, aged, and deformed $\mathrm{Fe}_{70} \mathrm{Mo}_{5} \mathrm{Ni}_{5} \mathrm{P}_{12.5} \mathrm{~B}_{2.5} \mathrm{C}_{5}$ (lines are guides to the eyes). Note that the $l_{C}$ were obtained using the correlation functions computed according to Eq. (15). in Figs. 7 and 8 strongly suggest that spin-misalignment correlations in the soft magnetic alloy are anisotropic and decay on a real-space length of the order of a few tens of nanometers. In this context, it is worth mentioning that an $\mathrm{x}$-ray and neutron-diffraction study on stress-relieved and field-annealed amorphous magnetic $\mathrm{Fe}_{78} \mathrm{Si}_{9} \mathrm{~B}_{13}$ ribbons [33] reports that modifications in the interatomic distances may greatly change the nature of the magnetic coupling, resulting in antiferromagnetic correlations that decay on an atomic scale.

\section{B. $\left(\mathrm{Nd}_{60} \mathrm{Fe}_{30} \mathrm{Al}_{10}\right)_{92} \mathrm{Ni}_{8}$}

In this subsection, we report and discuss only the main neutron results for the rare-earth-based hard magnetic BMG; we refer the reader to the supplemental material for further data [13].

The results of the magnetization measurements are shown in Fig. 9. Although an applied field of about $8 \mathrm{~T}$ is sufficient to bring the samples into the approach-to-saturation regime, complete saturation is not reached. The field dependence of the count rate follows the magnetization behavior, exhibiting a maximum at the respective coercive field $H_{c}$ : for the as-cast, aged, and deformed samples, the values of $H_{c}$ are, respectively, $0.30,0.34$, and $0.30 \mathrm{~T}$. We also would like to note that previous investigations on similar rare-earth-based magnetic BMGs reported a strong dependency of $H_{c}$ on the synthesis method and on the quenching rate [34]. The mechanism that leads to coercivity in these types of materials is generally believed to be due to the pinning of domain walls, for instance by 


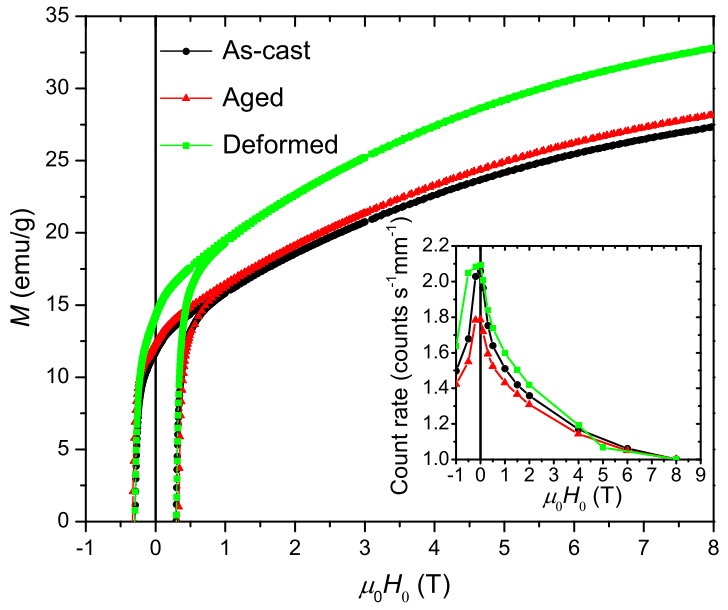

FIG. 9. Room-temperature magnetization curves of as-cast, aged, and deformed $\left(\mathrm{Nd}_{60} \mathrm{Fe}_{30} \mathrm{Al}_{10}\right)_{92} \mathrm{Ni}_{8}$ (only the upper quadrants are shown). The inset shows the field dependence of the neutron count rate of the samples normalized by the respective sample thickness. The measured $q$-range for the count rate is $0.035 \lesssim q \lesssim 0.3 \mathrm{~nm}^{-1}$.

paramagnetic Nd-rich precipitates that are present in the X-ray amorphous samples [35]. The most remarkable observation in Fig. 9 is, however, the increase of the magnetization on mechanical deformation, which is somewhat counterintuitive: application of mechanical load usually generates defects and/or disorder in the deformed material, and as a consequence one expects that the related spin misalignment in the vicinity of the defects contributes to a reduction of the magnetization component along the field direction [compare the $M\left(H_{0}\right)$ data of the soft magnetic BMG in Fig. 2]. At $8 \mathrm{~T}$, this increase of the magnetization amounts to $\sim 20 \%$ relative to the as-prepared sample. A possible explanation might be that through the plastic deformation, Nd-rich nanoclusters, which preexist in the amorphous sample [35], deform into aligned and shape-anisometric (e.g., platelet-shaped) nanocrystals, and that these contribute via their shape anisotropy to the magnetization. A similar increase of the magnetization was observed in plastically deformed Ba-ferrite composites [36]. Although our electron-microscopy data reveal crystalline regions in all three rare-earth-based specimens (see Fig. 7 in the Supplemental Material [13]), more work is certainly required to unambiguously determine the orientation relationships of the nanocrystals.

The total unpolarized scattering cross section of the hard magnetic BMG sample was measured in the magnetic-field range up to $8 \mathrm{~T}$ (see Figs. 1 and 2 in the Supplemental Material [13]). In contrast to $\mathrm{Fe}_{70} \mathrm{Mo}_{5} \mathrm{Ni}_{5} \mathrm{P}_{12.5} \mathrm{~B}_{2.5} \mathrm{C}_{5}$, where we observe a strong field dependency of $d \Sigma / d \Omega$ and of $d \Sigma_{M} / d \Omega$ by two orders of magnitude (see Fig. 5), the Nd-containing samples exhibit only a weak dependency of $d \Sigma / d \Omega$ on the applied magnetic field. The two-dimensional total scattering cross sections are isotropic for all applied field values investigated, which is likely related to the dominance of the isotropic nuclear scattering contribution, and they remain visually unchanged with decreasing magnetic field. On the other hand, when the scattering at the largest field of $8 \mathrm{~T}$ is subtracted from the data at lower fields, the resulting spin-misalignment scattering cross section $d \Sigma_{M} / d \Omega$ (see Fig. 10) is highly anisotropic. With the field decreasing from $8 \mathrm{~T}, d \Sigma_{M} / d \Omega$ exhibits an angular anisotropy that contains contributions due to a $\cos ^{2} \theta$ term (giving rise to a horizontally elongated pattern) and due to a $\sin ^{2} \theta \cos ^{2} \theta$ type term (yielding maxima roughly along the diagonals of the detector). With reference to the theoretical expressions for the spin-misalignment SANS cross section [Eqs. (6)-(10)], this suggests that in the hard magnetic BMG scattering contributions due to spatially fluctuating saturation magnetization [cf. the angular anisotropy of the response function $R_{M}$; Eq. (10)] play a significant role. This is in contrast to the soft magnetic BMG, where we have found that the term $S_{M} R_{M}$ is negligible and $d \Sigma_{M} / d \Omega$ is dominated by $S_{H} R_{H}$.

The anisotropic $d \Sigma_{M} / d \Omega$ translate into predominantly vertically aligned correlation functions $c(y, z)$ (see Fig. 6 in the Supplemental Material [13]); this is in agreement with the notion of spin-misalignment scattering originating from spin perturbations evolving in the plane perpendicular to the applied magnetic field. As an example, we display in Fig. 11 the field dependence of the azimuthally averaged correlation function of the deformed BMG sample. Increasing the field suppresses the amplitude as well as the range of gradients in the magnetic microstructure. The corresponding field-dependent correlation lengths (which are a measure for the magnitude of inhomogeneously magnetized regions) of all hard magnetic BMGs are shown in Fig. 12. The difference in $l_{C}$ between as-cast, aged, and deformed $\left(\mathrm{Nd}_{60} \mathrm{Fe}_{30} \mathrm{Al}_{10}\right)_{92} \mathrm{Ni}_{8}$ is only of the order of 2-3 nm; the $l_{C}$ values decrease from a maximum of $\sim 45 \mathrm{~nm}$ at the coercive field $\mu_{0} H_{c} \sim-0.3 \mathrm{~T}$ to $\sim 20 \mathrm{~nm}$ at $6 \mathrm{~T}$. The observation that the largest field dependencies of $d \Sigma / d \Omega$ and $d \Sigma_{M} / d \Omega$ are concentrated at the smallest $q$ (see Figs. 2 and 4 in the Supplemental Material [13]) indicates that significant magnetic scattering contributions originate from large-scale structures, which in future experiments may be probed by means of ultra-small-angle neutron scattering.

While the magnetization data (Fig. 9) show a strong dependency on the thermal and mechanical treatment, the SANS data (Figs. 10 and 12) are very similar for the as-cast, aged, and deformed states. This suggests that the microstructural changes that are responsible for $M\left(H_{0}\right)$ are not resolved by the SANS experiment (given the experimental $q_{\min }$ and $q_{\max }$ ), which probes a real-space structure between about 1 and 100 $\mathrm{nm}$; however, this observation does not imply that SANS is not suitable for resolving displacement fields in magnetic BMG in the given $q$-range. In this context, we refer to a recent SANS study that investigates the effect of the annealing conditions (heating rate and temperature) on the magnetic microstructure of sintered $\mathrm{Nd}-\mathrm{Fe}-\mathrm{B}$ magnets [37]; while the temperature treatment has a strong effect on the coercivity (with a reduction of about $50 \%$ upon annealing), the associated changes in the microstructure surprisingly do not show up (or at best show up only very weakly) in the neutron-scattering signal.

\section{SUMMARY AND CONCLUSIONS}

We have investigated the magnetic microstructure of soft magnetic $\mathrm{Fe}_{70} \mathrm{Mo}_{5} \mathrm{Ni}_{5} \mathrm{P}_{12.5} \mathrm{~B}_{2.5} \mathrm{C}_{5}$ and hard magnetic $\left(\mathrm{Nd}_{60} \mathrm{Fe}_{30} \mathrm{Al}_{10}\right)_{92} \mathrm{Ni}_{8}$ bulk metallic glasses (BMGs) in an asprepared, aged, and mechanically deformed state by means of 

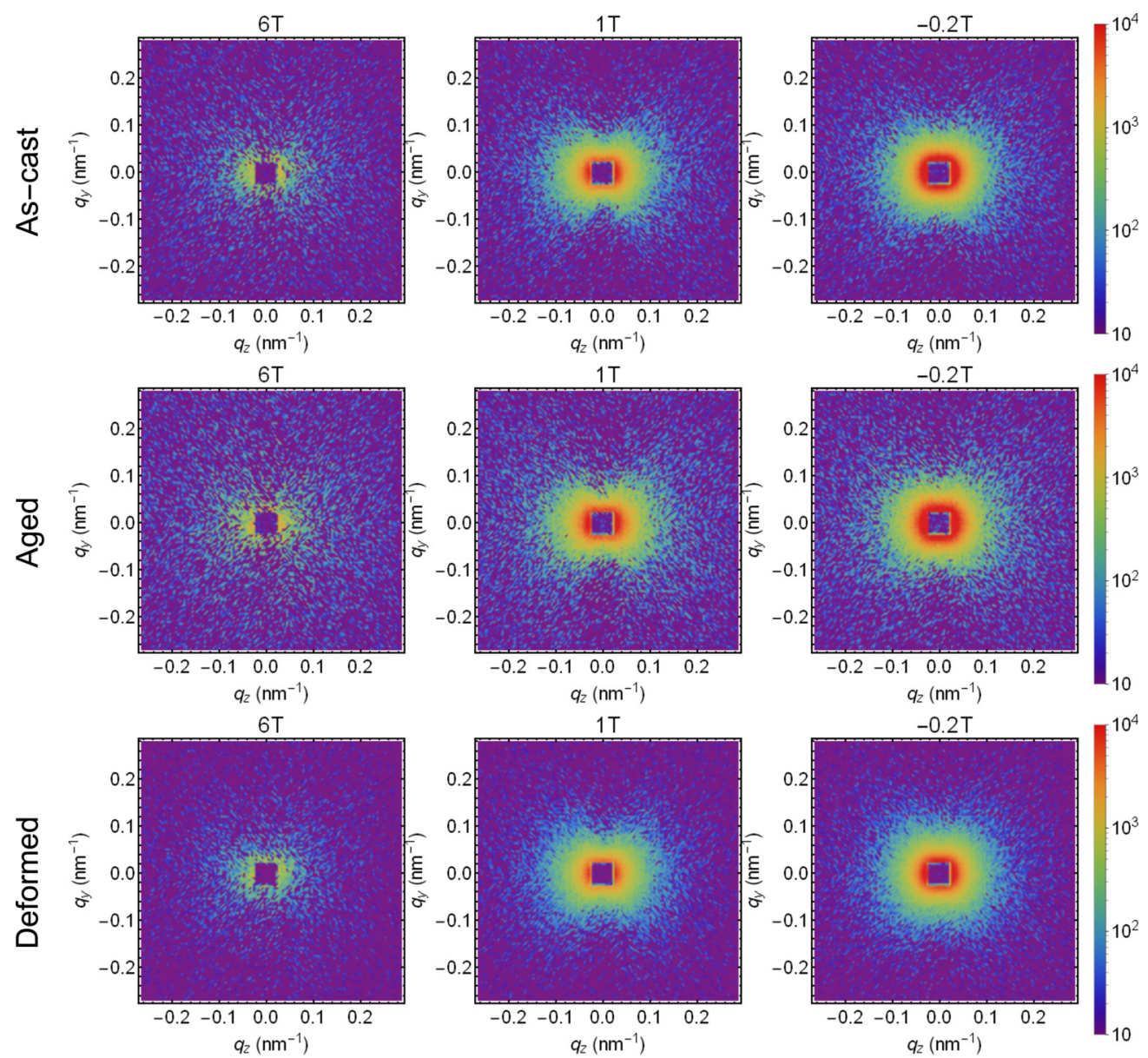

FIG. 10. Comparison of the two-dimensional spin-misalignment SANS cross sections $d \Sigma_{M} / d \Omega$ of as-cast, aged, and deformed $\left(\mathrm{Nd}_{60} \mathrm{Fe}_{30} \mathrm{Al}_{10}\right)_{92} \mathrm{Ni}_{8}$ at selected applied magnetic fields (logarithmic color scale). $d \Sigma / d \Omega$ at $8 \mathrm{~T}$ has been subtracted from each dataset. $\mathbf{H}_{0}$ is horizontal in the plane.

magnetic small-angle neutron scattering (SANS). Based on the analysis of the SANS cross section in terms of the correlation function of the spin misalignment, we could estimate the

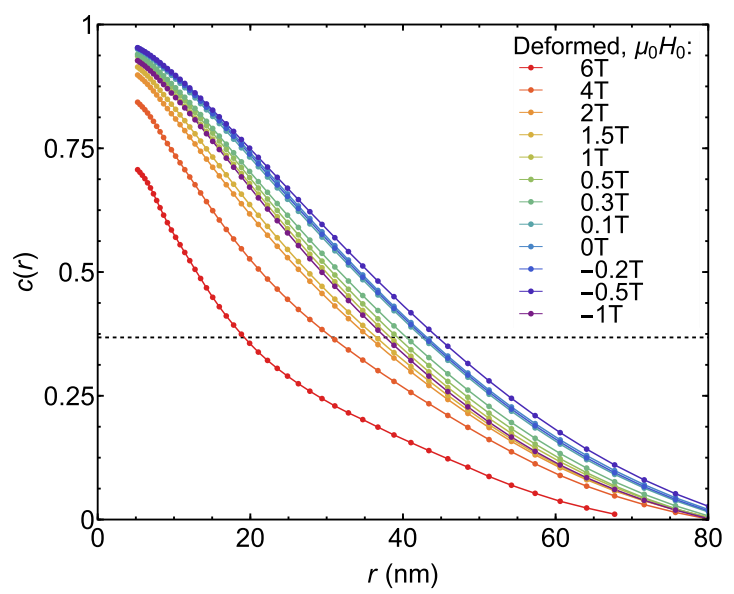

FIG. 11. Azimuthally averaged correlation function $c(r)$ of the deformed $\left(\mathrm{Nd}_{60} \mathrm{Fe}_{30} \mathrm{Al}_{10}\right)_{92} \mathrm{Ni}_{8}$ sample at selected applied magnetic fields (see the insets). Dashed horizontal line: $c(r)=\exp (-1)$. Note that the $c(r)$ were obtained by azimuthally averaging the twodimensional correlation functions [computed according to Eq. (14)]. characteristic length scale $l_{C}$, which provides a measure for the size of nonuniformly magnetized regions. For both sets of samples, we find magnetic-field-dependent $l_{C}$ values of the order of several tens of nanometers. From the comparison

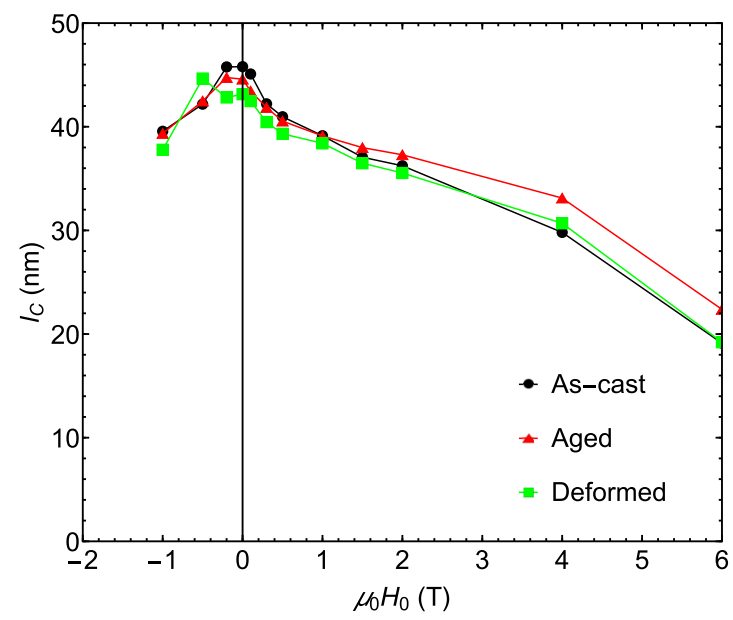

FIG. 12. Field dependence of the correlation length $l_{C}$ of as-cast, aged, and deformed $\left(\mathrm{Nd}_{60} \mathrm{Fe}_{30} \mathrm{Al}_{10}\right)_{92} \mathrm{Ni}_{8}$ (lines are guides to the eyes). The $l_{C}$ were computed using the $c(r)$ data displayed in Fig. 11 . 


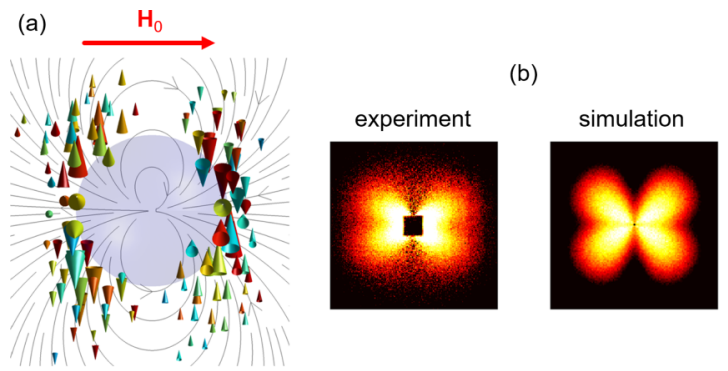

FIG. 13. (a) Computed spin misalignment (at $\mu_{0} H_{0}=0.6 \mathrm{~T}$ ) around a spherical pore (diameter: $12 \mathrm{~nm}$ ) in a ferromagnetic iron matrix (2D cut out of a 3D simulation). Shown is the magnetization component $\mathbf{M}_{\perp}(\mathbf{r})$ perpendicular to $\mathbf{H}_{0}$; the thickness of the arrows is proportional to the magnitude of $\mathbf{M}_{\perp}$. Solid gray lines: magnetodipolar field distribution. (b) Comparison between experimental SANS data [38] and micromagnetic simulations for the spin-misalignment SANS cross section of nanoporous iron. Images taken from Ref. [39].

of the experimentally observed angular anisotropy of the SANS cross section to theoretical predictions, we conclude that the magnetic microstructure of the soft magnetic BMG is dominated by magnetic anisotropy-field-related perturbations, while the mesoscale spin structure of the hard magnetic BMG is significantly influenced by magnetostatic fluctuations. For the rare-earth-containing deformed BMGs, we observe an unusual increase of high-field magnetization $(\sim 20 \%$ at $8 \mathrm{~T})$, which deserves further investigation.

We were not able in our feasibility study to resolve the nature of displacement fields generated by mechanical loading in magnetic BMGs. Nevertheless, the way seems paved for eventually elucidating this important open question by employing the spin degree of freedom of a magnetic material for decorating the displacement field. To solve this issue, we suggest the following approaches: (i) Future neutron work will concentrate on the in situ observation of the magnetic SANS cross section as a function of shear and/or tensile stress by analyzing the difference between loaded and unloaded states. Special attention will be paid to consistent sample preparation and generation of well-defined stress and strain states during deformation. Implementing these reasonable measures should enable one to disentangle spin-misalignment scattering due to spatial variations in the saturation magnetization from the strain-field-related scattering, which both result in clover-leaftype angular anisotropies with maxima along the detector diagonals. Moreover, the use of micromagnetic simulations for the modeling of displacement-field-induced spin disorder and the related magnetic SANS will further enhance our understanding of magnetic structure in BMG (see Fig. 13, where this approach has been employed in order to understand the spin structure of porous iron). (ii) Of course, the success of the in situ deformation and scattering experiment will crucially depend on sample quality; this entails the synthesis of high-quality homogeneous BMG and requires state-ofthe-art electron microscopy. (iii) By combining conventional small-angle neutron scattering with ultra-small-angle neutron scattering, one will be able to access smaller momentum transfers, and hence important missing information hidden by the beamstop in the conventional technique can be extracted. Likewise, the aid of polarized neutrons enables us to separate the nuclear coherent from the relevant magnetic scattering cross section.

\section{ACKNOWLEDGMENTS}

D.M. acknowledges financial support from the National Research Fund of Luxembourg (Grant No. INTER/DFG/12/07). This work is based on experiments performed at the Swiss spallation neutron source SINQ, Paul Scherrer Institute, Villigen, Switzerland and the Institute Laue-Langevin, Grenoble, France. We thank Jörg Schmauch (Universität des Saarlandes) for the electron-microscopy work and Marios Demetriou (Caltech) for providing the soft magnetic BMG.
[1] C. A. Schuh, T. C. Hufnagel, and U. Ramamurty, Mechanical behavior of amorphous alloys, Acta Mater. 55, 4067 (2007).

[2] Q. Luo and W. H. Wang, Rare earth based bulk metallic glasses, J. Non-Cryst. Solids 355, 759 (2009).

[3] C. Suryanarayana and A. Inoue, Iron-based bulk metallic glasses, Int. Mater. Rev. 58, 131 (2013).

[4] T. C. Hufnagel, C. A. Schuh, and M. L. Falk, Deformation of metallic glasses: Recent developments in theory, simulations, and experiments, Acta Mater. 109, 375 (2016).

[5] A. R. Hinkle, C. H. Rycroft, M. D. Shields, and M. L. Falk, Coarse graining atomistic simulations of plastically deforming amorphous solids, Phys. Rev. E 95, 053001 (2017).

[6] R. Dasgupta, H. G. E. Hentschel, and I. Procaccia, Microscopic Mechanism of Shear Bands in Amorphous Solids, Phys. Rev. Lett. 109, 255502 (2012).

[7] R. Dasgupta, H. G. E. Hentschel, and I. Procaccia, Yield strain in shear banding amorphous solids, Phys. Rev. E 87, 022810 (2013).
[8] K. E. Jensen, D. A. Weitz, and F. Spaepen, Local shear transformations in deformed and quiescent hard-sphere colloidal glasses, Phys. Rev. E 90, 042305 (2014).

[9] H. Kronmüller, A. Seeger, and M. Wilkens, Depolarisation und Kleinwinkelstreuung von Neutronen durch Gitterfehler in ferromagnetischen Kristallen, Z. Phys. 171, 291 (1963).

[10] G. Göltz, H. Kronmüller, A. Seeger, H. Scheuer, and W. Schmatz, Investigation of the dislocation arrangements in plastically deformed $\mathrm{Fe}$ single crystals using magnetic small-angle scattering of neutrons, Philos. Mag. A 54, 213 (1986).

[11] W. F. Brown, Jr., Theory of the approach to magnetic saturation, Phys. Rev. 58, 736 (1940).

[12] A. Michels, Magnetic small-angle neutron scattering of bulk ferromagnets, J. Phys.: Condens. Matter 26, 383201 (2014).

[13] See Supplemental Material at http://link.aps.org/supplemental/ 10.1103/PhysRevMaterials.1.074403 for further neutron and electron-microscopy data.

[14] A. Michels, P. Bender, R. Birringer, M. J. Deckarm, D. Honecker, A. Leibner, and D. Mettus, Imaging of displace- 
ment fields in a bulk amorphous alloy, Institut Laue-Langevin (ILL) (2015), http://doi.ill.fr/10.5291/ILL-DATA.5-32-816.

[15] C. D. Dewhurst, I. Grillo, D. Honecker, M. Bonnaud, M. Jacques, C. Amrouni, A. Perillo-Marcone, G. Manzin, and R. Cubitt, The small-angle neutron scattering instrument D33 at the Institut Laue-Langevin, J. Appl. Cryst. 49, 1 (2016).

[16] J. Kohlbrecher and W. Wagner, The new SANS instrument at the Swiss spallation source SINQ, J. Appl. Cryst. 33, 804 (2000).

[17] C. Dewhurst, Graphical reduction and analysis SANS program for MatlabTM, Institut Laue-Langevin, France, http://www.ill.fr/lss/grasp (2015).

[18] D. Honecker and A. Michels, Theory of magnetic small-angle neutron scattering of two-phase ferromagnets, Phys. Rev. B 87, 224426 (2013).

[19] A. Michels, D. Mettus, D. Honecker, and K. L. Metlov, Effect of Dzyaloshinski-Moriya interaction on elastic small-angle neutron scattering, Phys. Rev. B 94, 054424 (2016).

[20] H. Kronmüller and M. Fähnle, Micromagnetism and the Microstructure of Ferromagnetic Solids (Cambridge University Press, Cambridge, 2003).

[21] A. Guinier and G. Fournet, Small-Angle Scattering of X-rays (Wiley, New York, 1955).

[22] G. Porod, in Small Angle X-ray Scattering, edited by O. Glatter and O. Kratky (Academic, London, 1982), pp. 17-51.

[23] L. A. Feigin and D. I. Svergun, Structure Analysis by SmallAngle X-Ray and Neutron Scattering (Plenum, New York, 1987).

[24] G. Fritz and O. Glatter, Structure and interaction in dense colloidal systems: Evaluation of scattering data by the generalized indirect Fourier transformation method, J. Phys.: Condens. Matter 18, S2403 (2006).

[25] D. Mettus and A. Michels, Small-angle neutron scattering correlation functions of bulk magnetic materials, J. Appl. Cryst. 48, 1437 (2015).

[26] J.-P. Bick, D. Honecker, F. Döbrich, K. Suzuki, E. P. Gilbert, H. Frielinghaus, J. Kohlbrecher, J. Gavilano, E. M. Forgan, R. Schweins, P. Lindner, R. Birringer, and A. Michels, Magnetization reversal in Nd-Fe-B based nanocomposites as seen by magnetic small-angle neutron scattering, Appl. Phys. Lett. 102, 022415 (2013).

[27] O. Glatter, in Small-angle X-ray Scattering, edited by O. Glatter and O. Kratky (Academic, London, 1982), pp. 119-196.

[28] D. I. Svergun and M. H. J. Koch, Small-angle scattering studies of biological macromolecules in solution, Rep. Prog. Phys. 66, 1735 (2003).
[29] G. Fritz-Popovski, Two-dimensional indirect Fourier transformation for evaluation of small-angle scattering data of oriented samples, J. Appl. Cryst. 46, 1447 (2013).

[30] G. Fritz-Popovski, Interpretation of two-dimensional real-space functions obtained from small-angle scattering data of oriented microstructures, J. Appl. Cryst. 48, 44 (2015).

[31] P. Bender, L. K. Bogart, O. Posth, W. Szczerba, S. E. Rogers, A. Castro, L. Nilsson, L. J. Zeng, A. Sugunan, J. Sommertune, A. Fornara, D. González-Alonso, L. Fernández Barquín, and C. Johansson, Structural and magnetic properties of multi-core nanoparticles analysed using a generalised numerical inversion method, Sci. Rep. 7, 45990 (2017).

[32] E. A. Périgo, E. P. Gilbert, K. L. Metlov, and A. Michels, Experimental observation of magnetic poles inside bulk magnets via $\mathbf{q} \neq \mathbf{0}$ Fourier modes of magnetostatic field, New. J. Phys. 16, 123031 (2014).

[33] Q. A. Pankhurst, L. Fernández Barquín, J. D. Wicks, R. L. McGreevy, and M. R. J. Gibbs, Structural and magnetic anisotropy in amorphous alloy ribbons, J. Phys.: Condens. Matter 9, L375 (1997).

[34] R. S. Turtelli, D. Triyono, R. Grössinger, H. Michor, J. H. Espina, J. P. Sinnecker, H. Sassik, J. Eckert, G. Kumar, Z. G. Sun, and G. J. Fan, Coercivity mechanism in $\mathrm{Nd}_{60} \mathrm{Fe}_{30} \mathrm{Al}_{10}$ and $\mathrm{Nd}_{60} \mathrm{Fe}_{20} \mathrm{Co}_{10} \mathrm{Al}_{10}$ alloys, Phys. Rev. B 66, 054441 (2002).

[35] A. Bracchi, K. Samwer, P. Schaaf, J. F. Löffler, and S. Schneider, Phase separation and microstructure controlled magnetic properties of rapidly quenched $\mathrm{Nd}_{60} \mathrm{Fe}_{30} \mathrm{Al}_{10}$, Mater. Sci. Eng. A 375-377, 1027 (2004).

[36] Y. Suzuki, M. Awano, N. Kondo, and T. Ohji, Enhanced magnetization of $3 \mathrm{~mol} \%$ yttria-doped zirconia/barium hexaferrite by post-plastic deformation, J. Am. Ceram. Soc. 83, 1113 (2000).

[37] É. A. Périgo, I. Titov, R. Weber, D. Mettus, I. Peral, O. Vallcorba, D. Honecker, and A. Michels (unpublished).

[38] A. Michels, M. Elmas, F. Döbrich, M. Ames, J. Markmann, M. Sharp, H. Eckerlebe, J. Kohlbrecher, and R. Birringer, Porosity-induced spin disorder in nanocrystalline inert-gas-condensed iron, Europhys. Lett. 85, 47003 (2009).

[39] S. Erokhin, D. Berkov, and A. Michels, Dipolar spinmisalignment correlations in inhomogeneous magnets: Comparison between neutron scattering and micromagnetic approaches, Phys. Rev. B 92, 014427 (2015). 


\section{Supplemental Material to "Magnetic small-angle neutron scattering on bulk metallic glasses: a feasibility study for imaging displacement fields"}

Denis Mettus, ${ }^{1, *}$ Michael Deckarm, ${ }^{2}$ Andreas Leibner, ${ }^{2}$ Rainer Birringer, ${ }^{2, \dagger}$ Moritz Stolpe, ${ }^{3}$ Ralf Busch, ${ }^{3}$ Dirk Honecker, ${ }^{4}$ Joachim Kohlbrecher, ${ }^{5}$ Patrick Hautle, ${ }^{5}$ Nemanja Niketic, ${ }^{5}$ Jesús Rodríguez Fernández, ${ }^{6}$ Luis Fernández Barquín, ${ }^{6}$ and Andreas Michels ${ }^{1, \ddagger}$

${ }^{1}$ Physics and Materials Science Research Unit, University of Luxembourg, 162A Avenue de la Faïencerie, L-1511 Luxembourg, Grand Duchy of Luxembourg

${ }^{2}$ Universität des Saarlandes, Experimentalphysik Campus D2 2, D-66123, Saarbrücken, Germany

${ }^{3}$ Universität des Saarlandes, Chair of Metallic Materials, Department of Materials Science and Engineering Campus C6.3, D-66123 Saarbrücken, Germany

${ }^{4}$ Institut Laue-Langevin, 6 Rue Jules Horowitz, B.P. 156, F-38042 Grenoble Cedex 9, France

${ }^{5}$ Paul Scherrer Institute, CH-5232 Villigen PSI, Switzerland ${ }^{6}$ CITIMAC, Universidad de Cantabria, 39005 Santander, Spain

\section{Abstract}

Additional neutron and TEM data related to the above paper are displayed.

\footnotetext{
*denis.mettus@uni.lu

$\dagger$ r.birringer@nano.uni-saarland.de

$\ddagger$ andreas.michels@uni.lu
} 

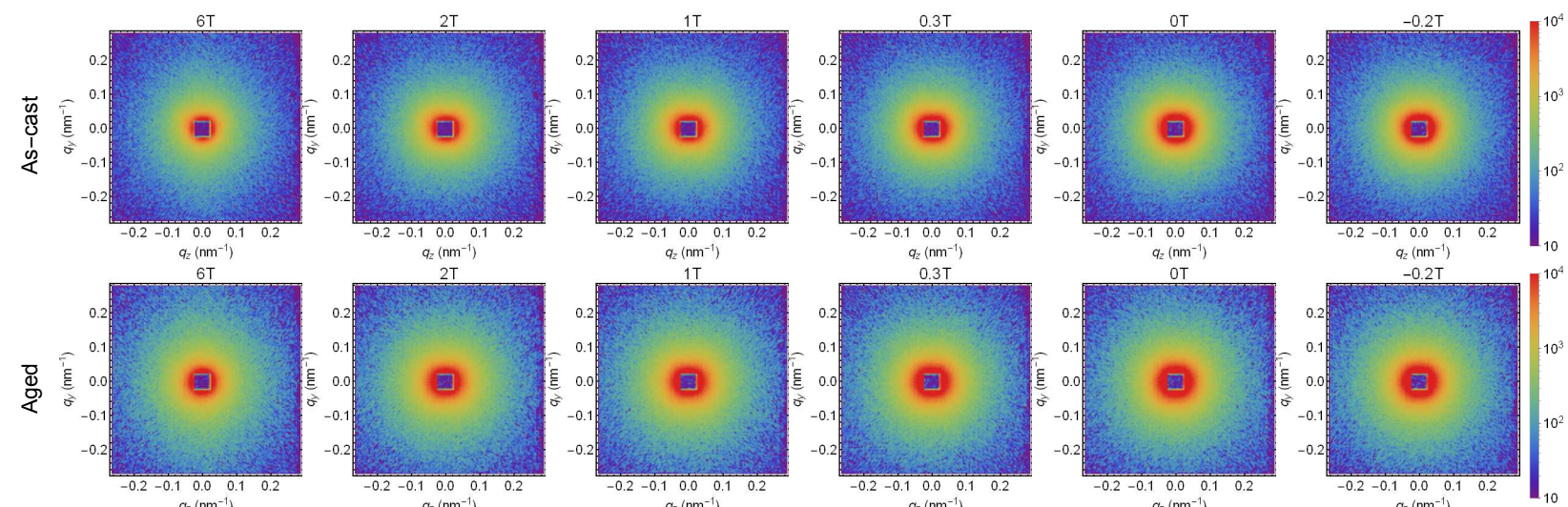

0.35

$q_{z}\left(\mathrm{~nm}^{-1}\right)$
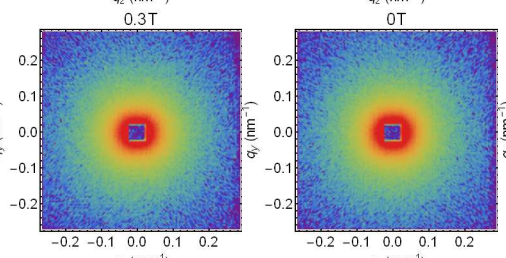

$q_{2}\left(\mathrm{~nm}^{-1}\right)$

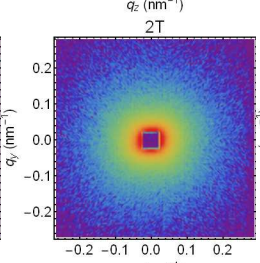

$q_{2}\left(\mathrm{~nm}^{-1}\right)$

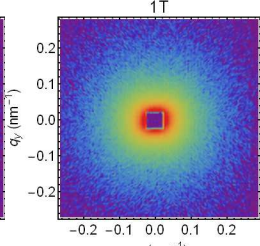

$\begin{array}{ccc}1 & 0.0 & 0.1 \\ Q_{2}\left(\mathrm{~nm}^{-1}\right)\end{array}$
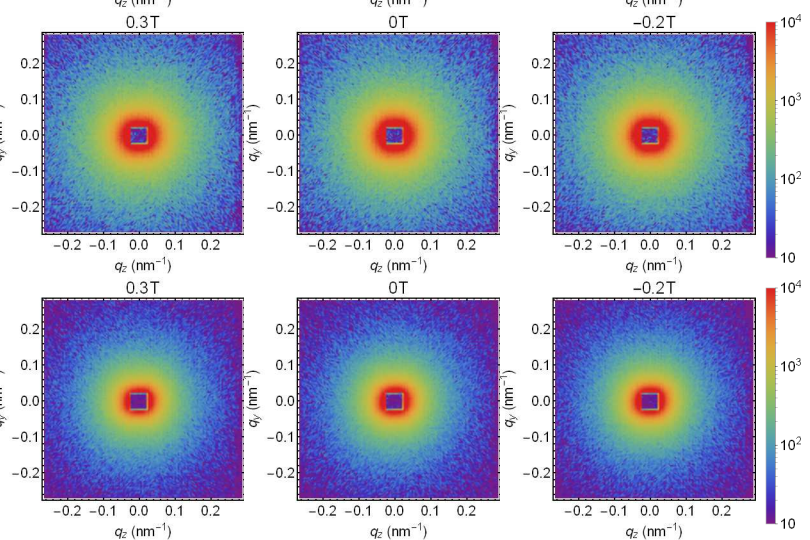

FIG. 1. Unpolarized (nuclear and magnetic) $d \Sigma / d \Omega$ of as-cast, aged, and deformed $\left(\mathrm{Nd}_{60} \mathrm{Fe}_{30} \mathrm{Al}_{10}\right)_{92} \mathrm{Ni}_{8}$ at selected applied magnetic fields (logarithmic color scale). $\mathbf{H}_{0}$ is horizontal in the plane.
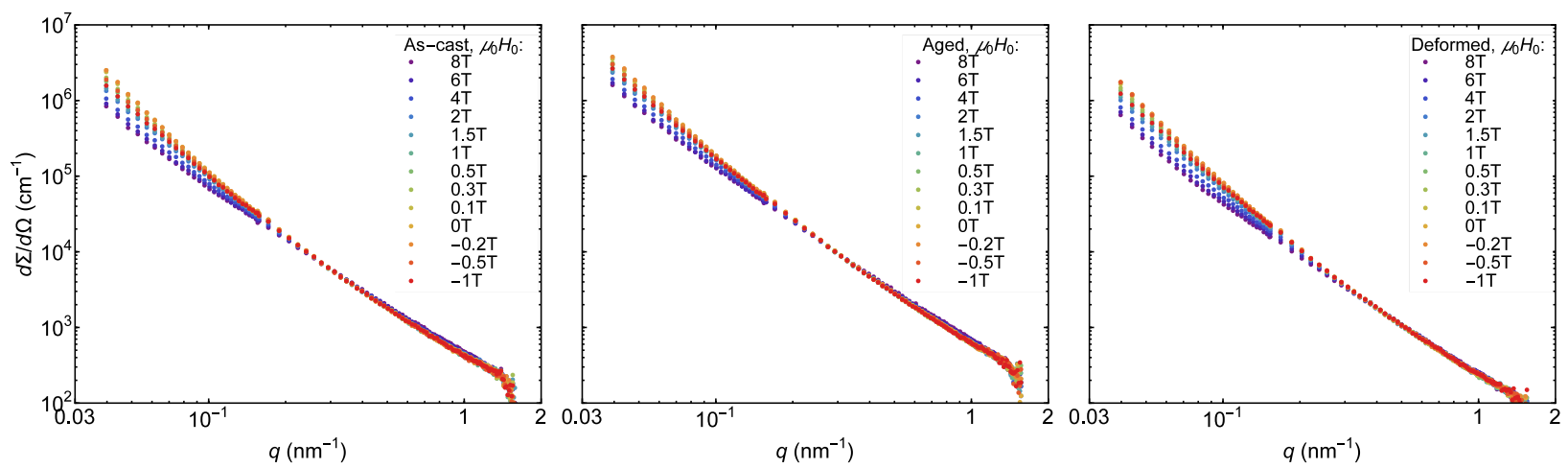

FIG. 2. Azimuthally-averaged total $d \Sigma / d \Omega$ of as-cast, aged, and deformed $\left(\mathrm{Nd}_{60} \mathrm{Fe}_{30} \mathrm{Al}_{10}\right)_{92} \mathrm{Ni}_{8}$ at selected applied magnetic fields (see insets, log-log scale). 

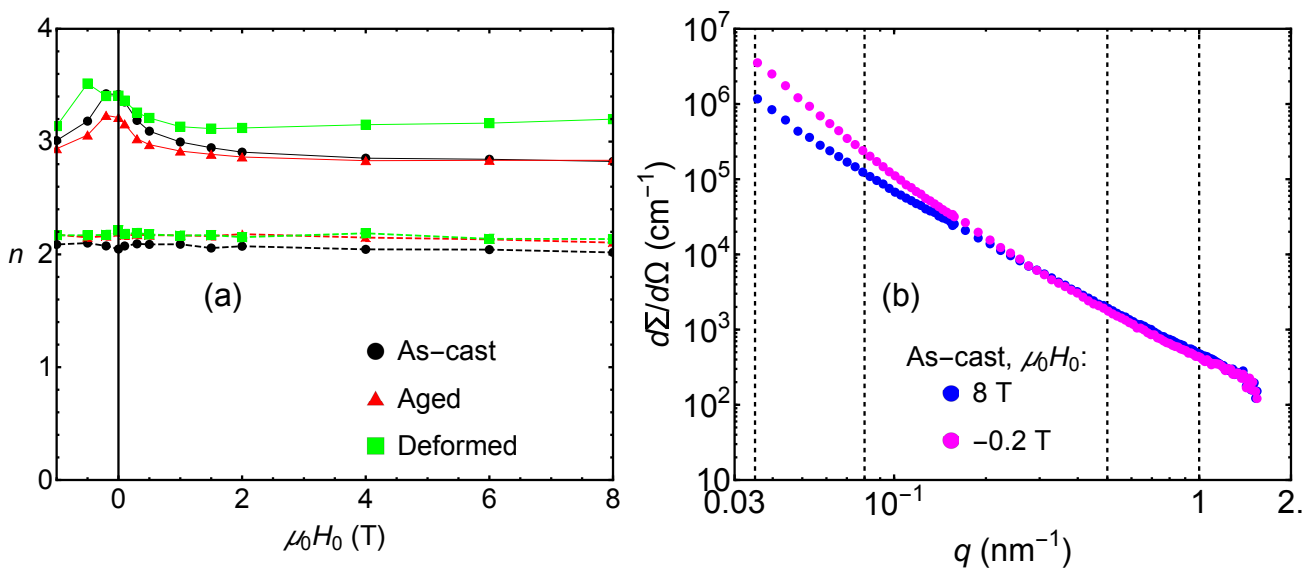

FIG. 3. (a) Field dependence of the power-law exponent $n$ in $d \Sigma / d \Omega=K / q^{n}$ for the total $d \Sigma / d \Omega$ of as-cast, aged, and deformed $\left(\mathrm{Nd}_{60} \mathrm{Fe}_{30} \mathrm{Al}_{10}\right)_{92} \mathrm{Ni}_{8}$. The dashed lines in (b) indicate the respective low- $q$ and high- $q$ ranges over which the fit was carried out. Solid (dashed) connecting lines in (a) correspond to the low- $q$ (high- $q$ ) region.
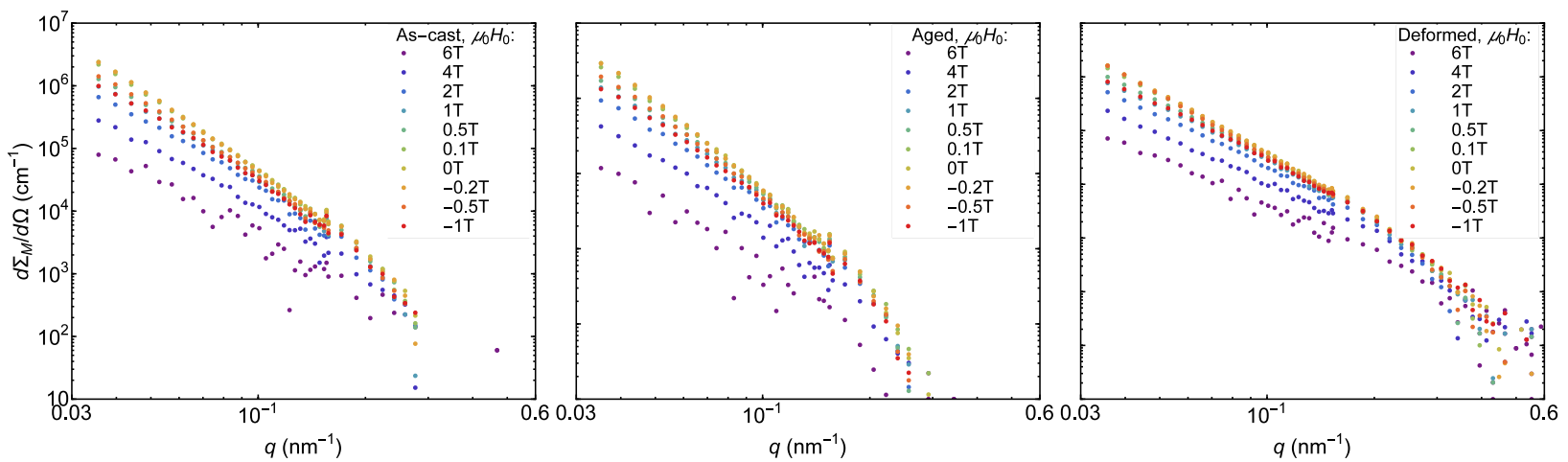

FIG. 4. Azimuthally-averaged spin-misalignment SANS cross section $d \Sigma_{M} / d \Omega$ of as-cast, aged, and deformed $\left(\mathrm{Nd}_{60} \mathrm{Fe}_{30} \mathrm{Al}_{10}\right)_{92} \mathrm{Ni}_{8}$ at selected applied magnetic fields (see insets, log-log scale).
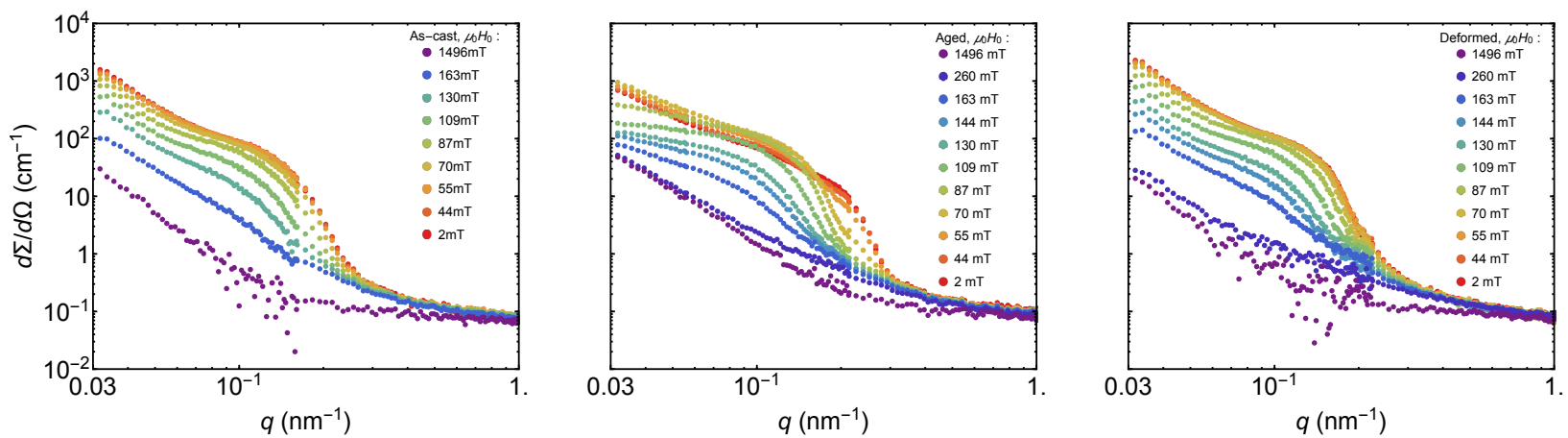

FIG. 5. Azimuthally-averaged total $d \Sigma / d \Omega$ of as-cast, aged, and deformed $\mathrm{Fe}_{70} \mathrm{Mo}_{5} \mathrm{Ni}_{5} \mathrm{P}_{12.5} \mathrm{~B}_{2.5} \mathrm{C}_{5}$ at selected applied magnetic fields (see insets, log-log scale). 

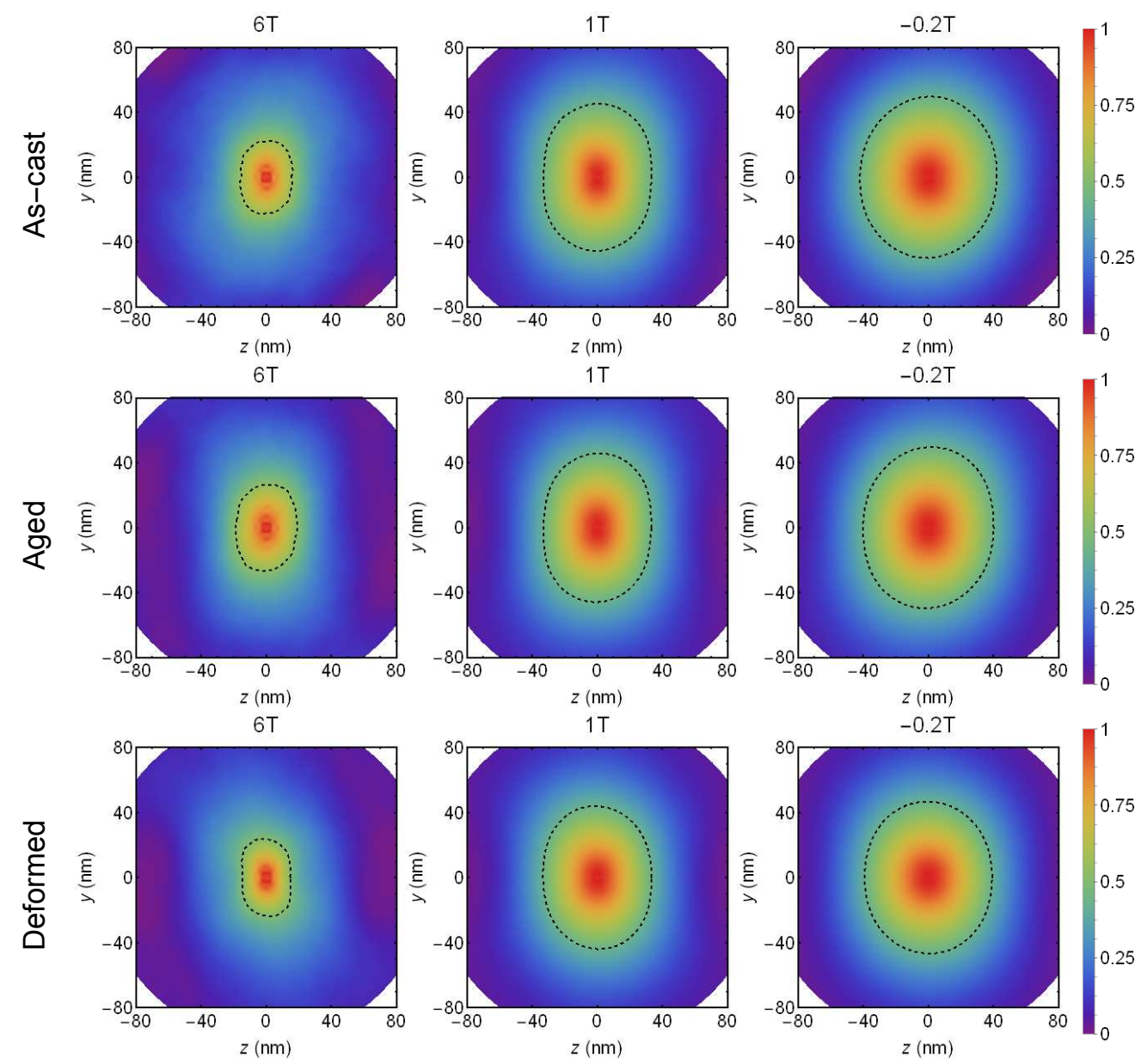

FIG. 6. Field dependence of the two-dimensional (normalized) correlation functions $c(y, z)$ of ascast, aged, and deformed $\left(\mathrm{Nd}_{60} \mathrm{Fe}_{30} \mathrm{Al}_{10}\right)_{92} \mathrm{Ni}_{8}$ at selected applied magnetic fields (see insets). $\mathbf{H}_{0}$ is horizontal in the plane. Dashed contour lines: $c(y, z)=\exp (-1)$.

As-cast

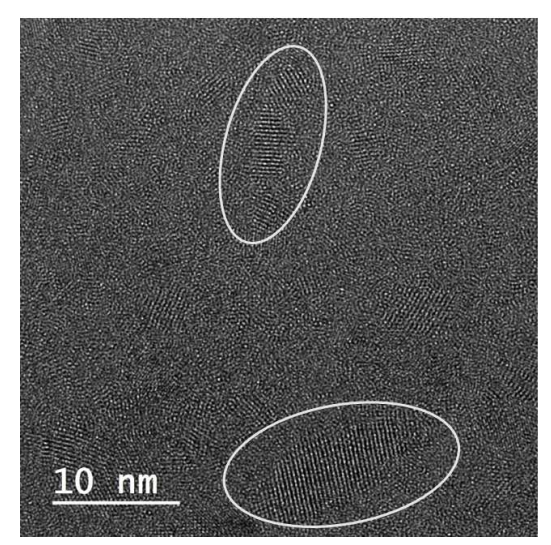

Aged

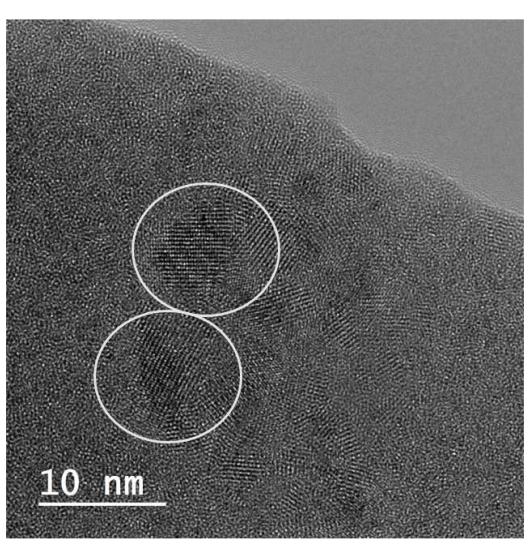

Deformed

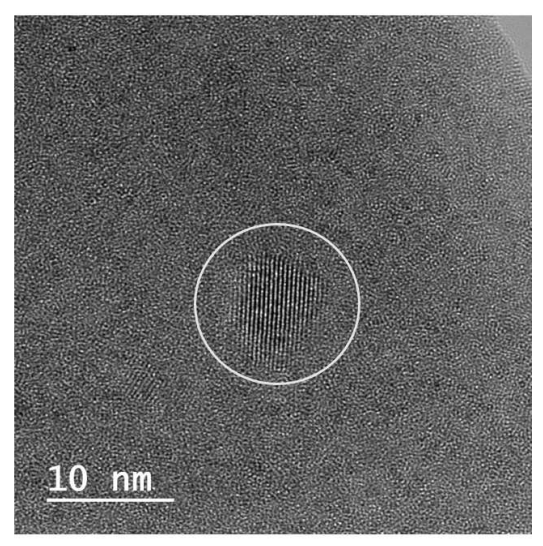

FIG. 7. TEM micrographs of as-cast, aged, and deformed $\left(\mathrm{Nd}_{60} \mathrm{Fe}_{30} \mathrm{Al}_{10}\right)_{92} \mathrm{Ni}_{8}$. 

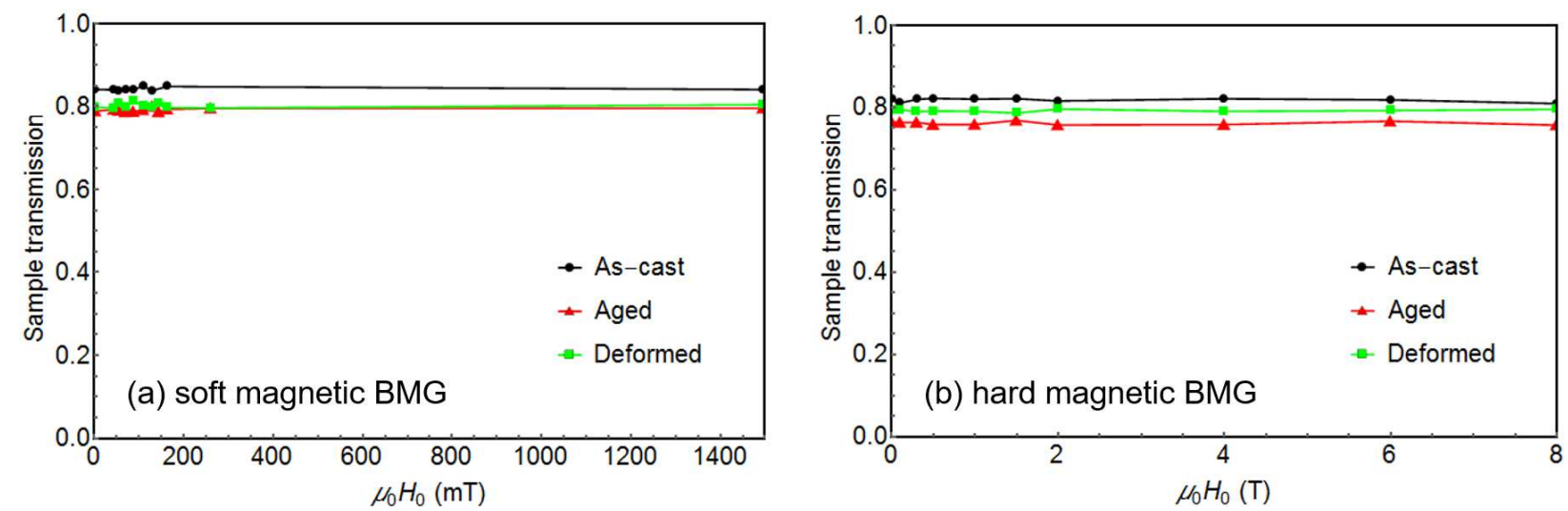

FIG. 8. Field dependence of the transmission of the (a) soft magnetic and (b) hard magnetic BMG. 